\title{
Synthesis and Conformational Studies of Dipeptides Constrained by Disubstituted 3-(Aminoethoxy)Propionic Acid Linkers
}

\author{
D. Srinivasa Reddy, David Vander Velde, and Jeffrey Aubé*
}

\section{Supporting Information}

\author{
Experimental Section
}

${ }^{1} \mathrm{H}$ and ${ }^{13} \mathrm{C}$-NMR Spectra of new compounds

Corresponding author:

Professor Jeffrey Aubé

Department of Medicinal Chemistry

1251 Wescoe Hall Drive, Room 4070

Malott Hall

University of Kansas

Lawrence, KS 66045-7582

Tel: 785.864 .4496

Fax: 785.864.5326

E-mail: jaube@ku.edu 


\section{Experimental Section:}

General methods have been published. ${ }^{1}$

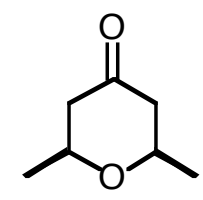

6

cis-2,6-Dimethyltetrahydropyran-4-one (6). ${ }^{2}$ A solution of 2,6-dimethylpyran-4-one (5.0 g, $40.3 \mathrm{mmol})$ in EtOH (30 mL), was stirred over 10\% Pd/C (0.5g) under hydrogen (30 Psi) for $8 \mathrm{~h}$. Catalyst was filtered off and concentrated. The crude product was purified on column chromatography (1:4 EtOAc/hexane) to furnish saturated ketone $\mathbf{6}(4.4 \mathrm{~g}, 85 \%)$ as an oil: IR (film) $1722 \mathrm{~cm}^{-1}$; ${ }^{1} \mathrm{H}$ NMR (500 MHz, $\left.\mathrm{CDCl}_{3}\right) \square 3.74(\mathrm{~m}, 2 \mathrm{H}), 2.34(\mathrm{~d}, J=14.6 \mathrm{~Hz}, 2 \mathrm{H}), 2.21(\mathrm{~m}$, 2H), $1.32(\mathrm{~d}, J=6.1 \mathrm{~Hz}, 6 \mathrm{H}) ;{ }^{13} \mathrm{C} \mathrm{NMR}\left(125.0 \mathrm{MHz}, \mathrm{CDCl}_{3}\right) \square 207.3,73.0,49.0,22.0$.

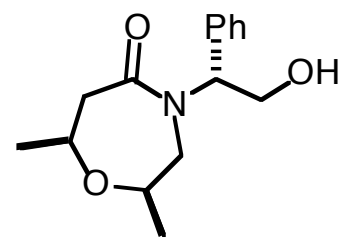

$8 a$

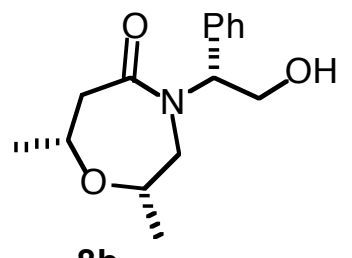

$8 b$

4-(2'-Hydroxy-1'R-phenylethyl)-2R,7S-dimethyl-[1,4]oxazepan-5-one (8a) and 4-(2'Hydroxy-1'R-phenylethyl)-2S,7R-dimethyl-[1,4]oxazepan-5-one (8b). To a solution of 6 (570 $\mathrm{mg}, 4.45 \mathrm{mmol})$ and $(R)$-2-azido-2-phenylethanol $7^{3}(500 \mathrm{mg}, 3.06 \mathrm{mmol})$ in $\mathrm{CCl}_{4}(10 \mathrm{~mL})$ was added $\mathrm{BF}_{3} \cdot \mathrm{OEt}_{2}(0.45 \mathrm{~mL}, 3.69 \mathrm{mmol})$ dropwise at $-15{ }^{\circ} \mathrm{C}$ and allowed to warm to room temperature. After stirring for $12 \mathrm{~h}$, solution was concentrated under reduced pressure, dissolved in THF $(10 \mathrm{~mL})$, and saturated $\mathrm{NaHCO}_{3}$ solution $(10 \mathrm{~mL})$ was added. The reaction was stirred for $1 \mathrm{~h}$ at room temperature, diluted with $\mathrm{CH}_{2} \mathrm{Cl}_{2}$, washed with water, dried $\left(\mathrm{Na}_{2} \mathrm{SO}_{4}\right)$, and concentrated to give a crude yellow oil. ${ }^{1} \mathrm{H}$ NMR analysis of the crude reaction mixture indicated an 88:12 ratio of diastereomers. Column chromatography (EtOAc $\square$ 1:20 $\mathrm{MeOH} / \mathrm{CH}_{2} \mathrm{Cl}_{2}$ ) 
afforded compounds 8a (562 $\mathrm{mg}$ ) and $\mathbf{8 b}(43 \mathrm{mg})$ in $75 \%$ combined yield. Major diastereomer (8a): oil; $[\square]_{\mathrm{D}}=-87.0\left(c 1.70, \mathrm{CHCl}_{3}\right)$; IR (film) $3404,1628 \mathrm{~cm}^{-1} ;{ }^{1} \mathrm{H}$ NMR (400 MHz, $\left.\mathrm{CDCl}_{3}\right) \square$ $7.24(\mathrm{~m}, 5 \mathrm{H}), 5.82(\mathrm{~m}, 1 \mathrm{H}), 4.08-3.90(\mathrm{~m}, 3 \mathrm{H}), 3.67(\mathrm{~m}, 1 \mathrm{H}), 3.31(\mathrm{dd}, J=8.2,18.6 \mathrm{~Hz}, 1 \mathrm{H})$, 2.96-2.79 (m, 3H), $2.47(\mathrm{~d}, J=14.9 \mathrm{~Hz}, 1 \mathrm{H}), 1.14(\mathrm{~d}, J=6.3 \mathrm{~Hz}, 3 \mathrm{H}), 0.73(\mathrm{~d}, J=6.4 \mathrm{~Hz}, 3 \mathrm{H})$; ${ }^{13} \mathrm{C}$ NMR $\left(100.6 \mathrm{MHz}, \mathrm{CDCl}_{3}\right) \square 175.3,137.7,129.0,128.7,128.4,75.9,71.6,61.0,58.4,51.6$, 47.7, 23.8, 20.3; MS (EI) m/e $264\left(\mathrm{M}^{+}+1\right)$; HRMS calcd for $\mathrm{C}_{15} \mathrm{H}_{22} \mathrm{NO}_{3}\left(\mathrm{M}^{+}+1\right): 264.1600$, found: 264.1595. Minor diastereomer (8b): oil; [D] $]_{\mathrm{D}}=-43.3\left(c\right.$ 1.76, $\left.\mathrm{CHCl}_{3}\right)$; IR (film) $3400,1629 \mathrm{~cm}^{-1}$; ${ }^{1} \mathrm{H}$ NMR $\left(400 \mathrm{MHz}, \mathrm{CDCl}_{3}\right) \square 7.42-7.28(\mathrm{~m}, 4 \mathrm{H}), 7.22(\mathrm{~d}, J=7.2 \mathrm{~Hz}, 1 \mathrm{H}), 5.89(\mathrm{dd}, J=4.8,9.0$ $\mathrm{Hz}, 1 \mathrm{H}), 4.21(\mathrm{dd}, J=4.7,11.5 \mathrm{~Hz}, 1 \mathrm{H}), 3.98-3.88(\mathrm{~m}, 2 \mathrm{H}), 3.77$ (pentet, $J=7.0 \mathrm{~Hz}, 1 \mathrm{H}), 3.20$ (dd, $J=8.2,16.0 \mathrm{~Hz}, 1 \mathrm{H}), 2.96-2.83(\mathrm{~m}, 3 \mathrm{H}), 2.60(\mathrm{~d}, J=14.7 \mathrm{~Hz}, 1 \mathrm{H}), 1.27(\mathrm{~d}, J=6.3 \mathrm{~Hz}, 3 \mathrm{H})$, $1.11(\mathrm{~d}, J=6.5 \mathrm{~Hz}, 3 \mathrm{H}) ;{ }^{13} \mathrm{C} \mathrm{NMR}\left(100.6 \mathrm{MHz}, \mathrm{CDCl}_{3}\right) \square$ 175.6, 137.3, 129.2, 128.2, 128.0, 75.8, 71.6, 62.5, 58.6, 52.5, 47.9, 23.9, 20.7; MS (EI) m/e $264\left(\mathrm{M}^{+}+1\right)$; HRMS calcd for $\mathrm{C}_{15} \mathrm{H}_{22} \mathrm{NO}_{3}\left(\mathrm{M}^{+}+1\right): 264.1600$, found 264.1604.

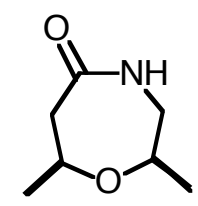

$(+)-9$

2R,7S-Dimethyl-[1,4]oxazepan-5-one ((+)-9). $N$-Substituted lactam 8a (550 mg, 2.08 mmol) in THF $(10 \mathrm{~mL})$ was added to refluxing ammonia (ca. $20 \mathrm{~mL})$ at $-78{ }^{\circ} \mathrm{C}$. Small sodium pieces were added to the mixture until it became deep blue in color. The reaction mixture was allowed to stir for 15 min before it was quenched with solid $\mathrm{NH}_{4} \mathrm{Cl}$. After the ammonia was evaporated, the residue was taken up in $\mathrm{CH}_{2} \mathrm{Cl}_{2}$ and washed with water and brine. The organic layer was dried over $\mathrm{Na}_{2} \mathrm{SO}_{4}$ and concentrated. The crude product was purified on column chromatography using EtOAc $\square$ 1:10 $\mathrm{MeOH} / \mathrm{CH}_{2} \mathrm{Cl}_{2}$ to afford lactam (+)-9 (264 mg) in 78\% 
yield. White crystalline solid; $\mathrm{mp} 144-145^{\circ} \mathrm{C}$; $[\square]_{\mathrm{D}}=+21.6\left(c 0.85, \mathrm{CHCl}_{3}\right)$, lit. $^{3}[\square]_{\mathrm{D}}=+22.6(c$ 1, $\mathrm{CHCl}_{3}$ ); IR (neat) 3214, $1676 \mathrm{~cm}^{-1} ;{ }^{1} \mathrm{H}$ NMR (400 MHz, $\left.\mathrm{CDCl}_{3}\right) \square 7.06$ (br s, 1H), 3.84 (m, 1H), $3.68(\mathrm{~m}, 1 \mathrm{H}), 3.32(\mathrm{ddd}, J=3.6,8.6,15.2 \mathrm{~Hz}, 1 \mathrm{H}), 2.98(\mathrm{dd}, J=7.7,15.4 \mathrm{~Hz}, 1 \mathrm{H}), 2.79$ $(\mathrm{dd}, J=9.7,15.0 \mathrm{~Hz}, 1 \mathrm{H}), 2.41(\mathrm{dd}, J=2.1,15.0 \mathrm{~Hz}, 1 \mathrm{H}), 1.25(\mathrm{~d}, J=6.3 \mathrm{~Hz}, 3 \mathrm{H}), 1.16(\mathrm{~d}, J=$ $8.0 \mathrm{~Hz}, 3 \mathrm{H}) ;{ }^{13} \mathrm{C}$ NMR $\left(100.6 \mathrm{MHz}, \mathrm{CDCl}_{3}\right) \square 177.3,76.9,71.7,50.0,47.5,23.9,20.3$; MS (EI) m/e $144\left(\mathrm{M}^{+}+1\right)$; HRMS calcd for $\mathrm{C}_{7} \mathrm{H}_{14} \mathrm{NO}_{2}\left(\mathrm{M}^{+}+1\right)$ : 144.1025 , found: 144.1014 .



Boc-Ala-Gly-(3S,5R-dimethyl-4-oxa-Aca)-OMe (10). The lactam (+)-9 (150 mg, 0.92 mmol) was dissolved in $6 \mathrm{~N} \mathrm{HCl}(5 \mathrm{~mL})$ and heated to reflux for $2 \mathrm{~h}$. After the solvent was removed in vacuo, the residue was dissolved in a minimum amount of $\mathrm{MeOH}$ and added to a prestirred mixture of $\mathrm{SOCl}_{2}(0.5 \mathrm{~mL})$ in $\mathrm{MeOH}(5 \mathrm{~mL})$ at $0{ }^{\circ} \mathrm{C}$. The mixture was stirred at room temperature overnight, and concentrated to obtain crude product that was used immediately in the next step without further purification.

To a suspension of BocAla-Gly-OH (246 mg, $1.0 \mathrm{mmol})$, EDCI $\bullet \mathrm{HCl}(192 \mathrm{mg}, 1.0$ $\mathrm{mmol})$, and $\mathrm{HOBt}(135 \mathrm{mg}, 1.0 \mathrm{mmol})$ in DCM $(10 \mathrm{~mL})$, the above compound $(0.92 \mathrm{mmol})$ and $\mathrm{Et}_{3} \mathrm{~N}(0.42 \mathrm{~mL}, 3.0 \mathrm{mmol})$ in $\mathrm{DMF}(2 \mathrm{~mL})$ was added at $0{ }^{\circ} \mathrm{C}$. The mixture was stirred at room temperature for $12 \mathrm{~h}$, at which time it was diluted with EtOAc and washed with saturated $\mathrm{NaHCO}_{3}, 10 \%$ citric acid, and brine. The organic layer was dried over $\mathrm{Na}_{2} \mathrm{SO}_{4}$ and concentrated. The crude product was purified by flash column chromatography to furnish title compound $\mathbf{1 0}$ (335 mg, 90\% after 3 steps). Oil; [ []$_{\mathrm{D}}=\square 24.2\left(c 1.07, \mathrm{CHCl}_{3}\right)$; IR (film) $3323,1660 \mathrm{~cm}^{-1} ;{ }^{1} \mathrm{H}$ NMR (400 MHz, $\left.\mathrm{CDCl}_{3}\right) \square 7.36$ (br s, 1H), 7.26 (br s, 1H), $5.50(\mathrm{~d}, J=6.8 \mathrm{~Hz}, 1 \mathrm{H}), 4.17$ (t, $J=$ $6.1 \mathrm{~Hz}, 1 \mathrm{H}), 4.03(\mathrm{dd}, J=5.9,16.6 \mathrm{~Hz}, 1 \mathrm{H}), 3.89(\mathrm{~m}, 1 \mathrm{H}), 3.82(\mathrm{dd}, J=4.8,16.8 \mathrm{~Hz}, 1 \mathrm{H}), 3.63$ 
(s, 3H), 3.54-3.44 (m, 2H), $2.82(\mathrm{~m}, 1 \mathrm{H}), 2.48-2.33(\mathrm{~m}, 2 \mathrm{H}), 1.35(\mathrm{~s}, 9 \mathrm{H}), 1.29(\mathrm{~d}, J=7.0 \mathrm{~Hz}$ $3 \mathrm{H}), 1.10(\mathrm{~d}, J=6.2 \mathrm{~Hz}, 3 \mathrm{H}), 1.03(\mathrm{~d}, J=6.1 \mathrm{~Hz}, 3 \mathrm{H}) ;{ }^{13} \mathrm{C} \mathrm{NMR}\left(100.6 \mathrm{MHz}, \mathrm{CDCl}_{3}\right) \square$ 173.6, $173.5,169.2,155.8,80.1,73.3,71.5,52.3,50.6,45.3,43.0,41.9,28.7,21.0,19.7,19.0$; MS (positive FABMS) $404\left(\mathrm{M}^{+}+1\right)$; HRMS calcd for $\mathrm{C}_{18} \mathrm{H}_{34} \mathrm{~N}_{3} \mathrm{O}_{7}\left(\mathrm{M}^{+}+1\right)$ : 404.2397, found: 404.2377.

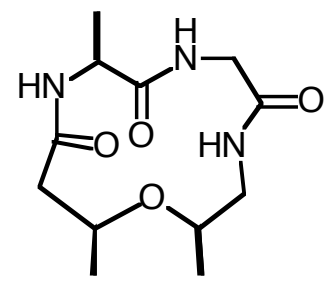

1

\section{General preparation of macrocycles: $2 R, 9 S, 13 S$-Trimethyl-1-oxa-4,7,10-triaza-}

cyclotridecane-5,8,11-trione (1). To a solution of the ester 10 (300 $\mathrm{mg} 0.74 \mathrm{mmol}$ ) in a minimum amount of THF was added $0.5 \mathrm{~N} \mathrm{LiOH}(4.4 \mathrm{~mL} 2.2 \mathrm{mmol})$ at $0{ }^{\circ} \mathrm{C}$. The mixture was stirred at room temperature for 2-3 $\mathrm{h}$, cooled to $0{ }^{\circ} \mathrm{C}$, and acidified carefully with $1 \mathrm{M} \mathrm{H}_{2} \mathrm{SO}_{4}$. The aqueous layer was extracted with EtOAc. The combined organic layers were dried over $\mathrm{Na}_{2} \mathrm{SO}_{4}$ and concentrated. The crude product was used in the next step without further purification. ${ }^{1} \mathrm{H}$ NMR (400 MHz, $\left.\mathrm{CDCl}_{3}\right) \square 7.25(\mathrm{~m}, 2 \mathrm{H}), 5.46(\mathrm{~d}, J=6.3 \mathrm{~Hz}, 1 \mathrm{H}), 4.50(\mathrm{br} \mathrm{s}, 1 \mathrm{H}), 4.29(\mathrm{dd}, J=$ 6.5, 16.5 Hz, 1H), $3.97(\mathrm{~m}, 1 \mathrm{H}), 3.83-3.58(\mathrm{~m}, 3 \mathrm{H}), 2.84(\mathrm{ddd}, J=3.5,8.4,13.2 \mathrm{~Hz}, 1 \mathrm{H}), 2.55-$ $2.46(\mathrm{~m}, 2 \mathrm{H}), 1.46(\mathrm{~s}, 9 \mathrm{H}), 1.38(\mathrm{~d}, J=6.9 \mathrm{~Hz}, 3 \mathrm{H}), 1.22(\mathrm{~d}, J=6.3 \mathrm{~Hz}, 3 \mathrm{H}), 1.16(\mathrm{~d}, J=6.2 \mathrm{~Hz}$, $3 \mathrm{H})$.

The above material (185 mg, $0.46 \mathrm{mmol})$ was dissolved in a 1:1 mixture of trifluoroacetic acid and DCM, and the solution was stirred for $1-2 \mathrm{~h}$ at room temperature. All volatiles were evaporated and the residue dried under vacuum. The crude product was dissolved in DMF (92 $\mathrm{mL}, 0.005 \mathrm{M}), \mathrm{NaHCO}_{3}(155 \mathrm{mg}, 1.85 \mathrm{mmol})$ followed by DECP $(0.3 \mathrm{~mL}, 1.85 \mathrm{mmol})$ were 
added at $0{ }^{\circ} \mathrm{C}$. After stirring $20 \mathrm{~h}$ at room temperature, DMF was removed under vacuum keeping the temperature below $50{ }^{\circ} \mathrm{C}$. The residue was purified by column chromatography $(1: 20$ 1:10 MeOH/ $\left.\mathrm{CH}_{2} \mathrm{Cl}_{2}\right)$ to furnish cyclic peptide $\mathbf{1}$ as white solid $(98 \mathrm{mg})$ in $51 \%$ yield starting from ester 10. White solid, $\mathrm{mp} 258^{\circ} \mathrm{C}(\mathrm{dec})$; [ []$_{\mathrm{D}}=-16.2(c 0.54, \mathrm{MeOH})$; IR (film) 3310, 1682, $1638 \mathrm{~cm}^{-1} ;{ }^{1} \mathrm{H}$ NMR $\left(500 \mathrm{MHz}, \mathrm{CDCl}_{3}\right) \square 8.60(\mathrm{~d}, J=6.7 \mathrm{~Hz}, 1 \mathrm{H}), 8.49(\mathrm{t}, J=6.2 \mathrm{~Hz}, 1 \mathrm{H}), 6.79$ $(\mathrm{t}, J=4.9 \mathrm{~Hz}, 1 \mathrm{H}), 4.22($ pentet, $J=6.9 \mathrm{~Hz}, 1 \mathrm{H}), 3.96(\mathrm{~m}, 1 \mathrm{H}), 3.78(\mathrm{dd}, J=5.6,16.7 \mathrm{~Hz}, 1 \mathrm{H})$, $3.61(\mathrm{~m}, 1 \mathrm{H}), 3.46-3.41(\mathrm{~m}, 1 \mathrm{H}), 3.13-3.05(\mathrm{~m}, 1 \mathrm{H}), 2.97-2.93(\mathrm{~m}, 1 \mathrm{H}), 2.34-2.24(\mathrm{~m}, 2 \mathrm{H}), 1.20$ $(\mathrm{d}, J=7.0 \mathrm{~Hz}, 3 \mathrm{H}), 1.13(\mathrm{~d}, J=6.0 \mathrm{~Hz}, 3 \mathrm{H}), 1.00(\mathrm{~d}, J=6.2 \mathrm{~Hz}, 3 \mathrm{H}) ;{ }^{13} \mathrm{C}$ NMR $(100.6 \mathrm{MHz}$ $\left.\mathrm{CDCl}_{3}\right) \square$ 173.4, 172.8, 169.5, 73.4, 71.6, 50.0, 44.1, 43.3, 43.2, 21.7, 20.5, 15.9; MS (positive FABMS) $272\left(\mathrm{M}^{+}+1\right)$; HRMS calcd for $\mathrm{C}_{12} \mathrm{H}_{22} \mathrm{~N}_{3} \mathrm{O}_{4}\left(\mathrm{M}^{+}+1\right)$ : 272.1610, found: 272.1603.

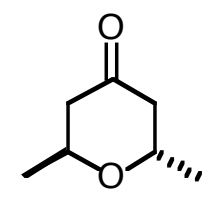

12

( \pm )-trans-2,6-Dimethyltetrahydropyran-4-one (12). ${ }^{4}$ To a suspension of CuI $(6.1 \mathrm{~g}$, $32.0 \mathrm{mmol})$ in ether $(50 \mathrm{~mL})$ was added a $1.4 \mathrm{M}$ solution of MeLi $(34 \mathrm{~mL}, 48.0 \mathrm{mmol})$ at $0{ }^{\circ} \mathrm{C}$. After $15 \mathrm{~min}$, enone $11(1.8 \mathrm{~g}, 16.1 \mathrm{mmol})^{5}$ in ether $(20 \mathrm{~mL})$ was added slowly at $0{ }^{\circ} \mathrm{C}$ for 10 min. The reaction mixture was warmed up to room temperature and allowed to stir for $2 \mathrm{~h}$, then quenched with saturated $\mathrm{NH}_{4} \mathrm{Cl}$ solution and extracted with ether. The combined organic layers were dried over $\mathrm{MgSO}_{4}$, filtered, and concentrated to furnish $12(1.79 \mathrm{~g}, 87 \%)$ as an oil. IR (film) $1720 \mathrm{~cm}^{-1} ;{ }^{1} \mathrm{H}$ NMR $\left(400 \mathrm{MHz}, \mathrm{CDCl}_{3}\right) \square 4.28(\mathrm{~m}, 2 \mathrm{H}), 2.50(\mathrm{dd}, J=4.4,14.1 \mathrm{~Hz}, 2 \mathrm{H}), 2.19(\mathrm{dd}$, $J=6.5,14.1 \mathrm{~Hz}, 2 \mathrm{H}), 1.23(\mathrm{~d}, J=6.5 \mathrm{~Hz}, 6 \mathrm{H}) ;{ }^{13} \mathrm{C} \mathrm{NMR}\left(100.6 \mathrm{MHz}, \mathrm{CDCl}_{3}\right) \square 208.0,68.6$, 48.6, 20.9. 


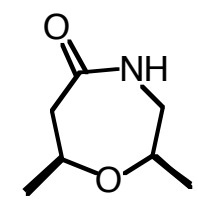

$( \pm)-9$

(士)-2,7-cis-Dimethyl-[1,4]oxazepan-5-one ((士)-9). $\mathrm{Na}_{2} \mathrm{CO}_{3}(3.72 \mathrm{~g}, 35.1 \mathrm{mmol})$ and $\mathrm{NH}_{2} \mathrm{OH} \cdot \mathrm{HCl}(2.44 \mathrm{~g}, 35.1 \mathrm{mmol})$ were dissolved in water $(30 \mathrm{~mL})$ and cooled in an ice bath. Pyrone $6(1.50 \mathrm{~g}, 11.7 \mathrm{mmol})$ in ether $(60 \mathrm{~mL})$ was added and the reaction allowed to stir for $2 \mathrm{~h}$ at $0{ }^{\circ} \mathrm{C}$. The reaction mixture was partitioned and the aqueous layer extracted with ether. The combined organic layers were washed with water and dried $\left(\mathrm{Na}_{2} \mathrm{SO}_{4}\right)$. The residue obtained after the evaporation of solvent was heated in ca. $5 \mathrm{~g}$ of PPA at $110^{\circ} \mathrm{C}$ for $15 \mathrm{~min}$. The reaction mixture was cooled to room temperature and poured into crushed ice with vigorous stirring, extracted with $\mathrm{CHCl}_{3}$, washed, and dried $\left(\mathrm{Na}_{2} \mathrm{SO}_{4}\right)$. Column chromatography (EtOAc $\square$ 1:20 $\left.\mathrm{MeOH} / \mathrm{CH}_{2} \mathrm{Cl}_{2}\right)$ afforded lactam ( \pm )-9 (1.05 g, 55\%) as white solid; mp 126-127 ${ }^{\circ} \mathrm{C}$. All other spectral data is same as enantiomerically pure lactam $(+)-9$.<smiles>COC(=O)C[C@H](C)O[C@H](C)CNC(=O)CNC(=O)C(C)NC(=O)OCc1ccccc1</smiles>

10

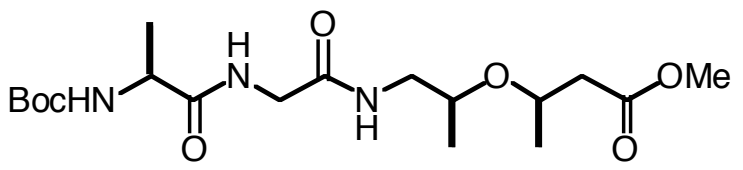

14

Boc-Ala-Gly-(3S,5R-dimethyl-4-oxa-Aca)-OMe (10) and Boc-Ala-Gly-(3R,5S-

dimethyl-4-oxa-Aca)-OMe (14). Isolated as a 1:1 mixture of diastereomers. IR (film) 3323, $1667 \mathrm{~cm}^{-1} ;{ }^{1} \mathrm{H}$ NMR (400 MHz, $\mathrm{CDCl}_{3}$ ) 77.40 (br s, 2H), 6.93 (br s, 2H), 5.16 (br s, 2H), 4.283.85 (series of m, 8H), $3.75(\mathrm{~s}, 6 \mathrm{H}), 3.73-3.50(\mathrm{~m}, 4 \mathrm{H}), 2.93(\mathrm{~m}, 2 \mathrm{H}), 2.54-2.46(\mathrm{~m}, 4 \mathrm{H}), 1.46(\mathrm{~s}$, 18H), $1.39(\mathrm{~d}, J=7.0 \mathrm{~Hz}, 6 \mathrm{H}), 1.19(\mathrm{~d}, J=6.1 \mathrm{~Hz}, 6 \mathrm{H}), 1.14(\mathrm{~d}, J=6.2 \mathrm{~Hz}, 6 \mathrm{H})$; MS (positive FABMS $404\left(\mathrm{M}^{+}+1\right)$; HRMS calcd for $\mathrm{C}_{18} \mathrm{H}_{34} \mathrm{~N}_{3} \mathrm{O}_{7}\left(\mathrm{M}^{+}+1\right)$ : 404.2397, found: 404.2397 . 


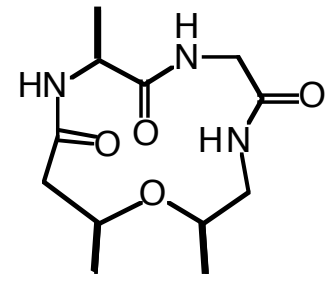

1

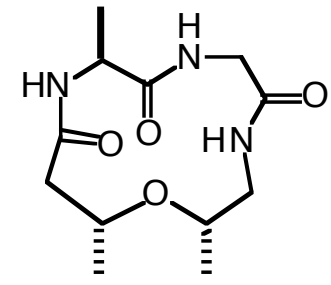

2

2R,9S,13S-Trimethyl-1-oxa-4,7,10-triaza-cyclotridecane-5,8,11-trione (1) and 2S,9S,13R-Trimethyl-1-oxa-4,7,10-triaza-cyclotridecane-5,8,11-trione (2). Prepared (48\% combined yield) as described for $\mathbf{1}$ and were separated by column chromatography (1:20 1:10 $\mathrm{MeOH}-\mathrm{CH}_{2} \mathrm{Cl}_{2}$ ). Compound 1 gave data as reported above. Compound 2: white solid, mp $230{ }^{\circ} \mathrm{C}$ (dec); $[\square]_{\mathrm{D}}=-15.9$ ( $\left.0.32, \mathrm{MeOH}\right) ; \mathrm{IR}(\mathrm{KBr}) 3316,1687,1640 \mathrm{~cm}^{-1} ;{ }^{1} \mathrm{H}$ NMR (500 MHz, $\left.\mathrm{CDCl}_{3}\right) \square 8.52(\mathrm{~d}, J=6.5 \mathrm{~Hz}, 1 \mathrm{H}), 8.33(\mathrm{t}, J=6.1 \mathrm{~Hz}, 1 \mathrm{H}), 6.81(\mathrm{~d}, J=7.3 \mathrm{~Hz}, 1 \mathrm{H}), 4.03(\mathrm{~m}$, 1H), $3.75(\mathrm{~m}, 2 \mathrm{H}), 3.49(\mathrm{~m}, 2 \mathrm{H}), 2.54(\mathrm{~m}, 1 \mathrm{H}), 2.35(\mathrm{~d}, J=12.0 \mathrm{~Hz}, 2 \mathrm{H}), 2.07(\mathrm{dd}, J=10.3,13.3$ $\mathrm{Hz}, 1 \mathrm{H}) 1.22(\mathrm{~d}, J=7.0 \mathrm{~Hz}, 3 \mathrm{H}), 1.13(\mathrm{~d}, J=6.1 \mathrm{~Hz}, 3 \mathrm{H}), 1.07(\mathrm{~d}, J=6.1 \mathrm{~Hz}, 3 \mathrm{H}) ;{ }^{13} \mathrm{C}$ NMR $\left(100.6 \mathrm{MHz}, \mathrm{CDCl}_{3}\right) \square$ 172.6, 172.5, 169.3, 76.1, 75.1, 50.1, 45.3, 44.4, 43.3, 23.2, 21.0, 16.6; MS (positive FABMS) $272\left(\mathrm{M}^{+}+1\right)$; HRMS calcd for $\mathrm{C}_{12} \mathrm{H}_{22} \mathrm{~N}_{3} \mathrm{O}_{4}\left(\mathrm{M}^{+}+1\right)$ : 272.1610, found: 272.1589 .

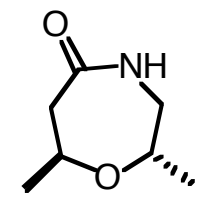

$( \pm)-13$

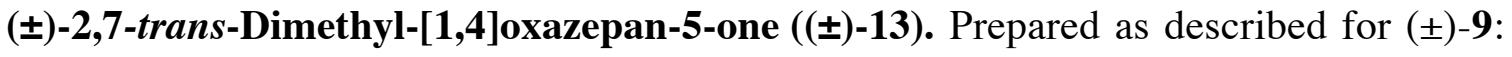
mp 76-77 ${ }^{\circ} \mathrm{C}$; IR (film) 3248, $1667 \mathrm{~cm}^{-1} ;{ }^{1} \mathrm{H}$ NMR (400 MHz, $\mathrm{CDCl}_{3}$ ) 66.87 (br s, 1H), 4.15 (m, 1H), $4.03(\mathrm{~m}, 1 \mathrm{H}), 3.33(\mathrm{dd}, J=6.1,15.3 \mathrm{~Hz}, 1 \mathrm{H}), 3.17(\mathrm{~m}, 1 \mathrm{H}), 2.77(\mathrm{dd}, J=1.6,14.8 \mathrm{~Hz}, 1 \mathrm{H})$, $2.56(\mathrm{dd}, J=7.2,14.7 \mathrm{~Hz}, 1 \mathrm{H}), 1.28(\mathrm{~d}, J=6.6 \mathrm{~Hz}, 3 \mathrm{H}), 1.19(\mathrm{~d}, J=6.5 \mathrm{~Hz}, 3 \mathrm{H}) ;{ }^{13} \mathrm{C}$ NMR 
(100.6 MHz, $\left.\mathrm{CDCl}_{3}\right) \square 176.6,69.4,65.4,49.1,46.0,20.2,17.7$; MS (EI) m/e 144 (M+1); HRMS calcd for $\mathrm{C}_{7} \mathrm{H}_{14} \mathrm{NO}_{2}\left(\mathrm{M}^{+}+1\right)$ : 144.1025 , found: 144.1021 .<smiles>COC(=O)CC(C)O[C@H](C)CNC(=O)CNC(=O)C(C)NC(=O)OCc1ccccc1</smiles>

15<smiles>COC(=O)C[C@H](C)OC(C)CNC(=O)CNC(=O)C(C)NC(=O)OCc1ccccc1</smiles>

16

Boc-Ala-Gly-(3R,5R-dimethyl-4-oxa-Aca)-OMe (15) and Boc-Ala-Gly-(3S,5S-

dimethyl-4-oxa-Aca)-OMe (16). Isolated as a 1:1 mixture of diastereomers. IR (film) 3316, $1659 \mathrm{~cm}^{-1} ;{ }^{1} \mathrm{H}$ NMR (400 MHz, $\mathrm{CDCl}_{3}$ ) $\mathrm{N} 7.24$ (br s, 2H), 6.76 (br s, 2H), 5.41 (br s, 2H), 4.19 $(\mathrm{m}, 2 \mathrm{H}), 4.00-3.83(\mathrm{~m}, 6 \mathrm{H}), 3.65(\mathrm{~s}, 6 \mathrm{H}), 3.65-3.57(\mathrm{~m}, 2 \mathrm{H}), 3.43-3.31(\mathrm{~m}, 2 \mathrm{H}), 3.10-2.98(\mathrm{~m}$, 2H), 2.47 (ddd, $J=4.4,8.0,12.4 \mathrm{~Hz}, 2 \mathrm{H}), 2.36(\mathrm{dd}, J=3.8,15.0 \mathrm{~Hz}, 2 \mathrm{H}), 1.41(\mathrm{~s}, 18 \mathrm{H}), 1.35$ (d, $J=7.0 \mathrm{~Hz}, 6 \mathrm{H}), 1.14(\mathrm{~d}, J=6.1 \mathrm{~Hz}, 6 \mathrm{H}), 1.05(\mathrm{~d}, J=6.1 \mathrm{~Hz}, 6 \mathrm{H})$; MS (positive FABMS) 404 $\left(\mathrm{M}^{+}+1\right)$; HRMS calcd for $\mathrm{C}_{18} \mathrm{H}_{34} \mathrm{~N}_{3} \mathrm{O}_{7}\left(\mathrm{M}^{+}+1\right)$ : 404.2397, found: 404.2393.

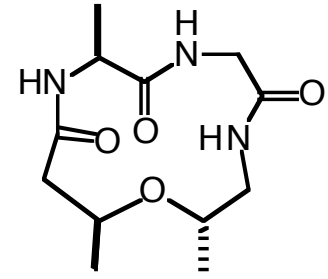

3

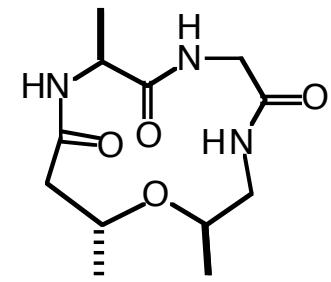

4

2S,9S,13S-Trimethyl-1-oxa-4,7,10-triaza-cyclotridecane-5,8,11-trione (3) and 2R,9S,13R-Trimethyl-1-oxa-4,7,10-triaza-cyclotridecane-5,8,11-trione (4). Compound 3 : white solid, mp $228{ }^{\circ} \mathrm{C}(\mathrm{dec})$; [ []$_{\mathrm{D}}=-98.4\left(c\right.$ 0.67, MeOH); IR (KBr) 3275, 1674, $1640 \mathrm{~cm}^{-1} ;{ }^{1} \mathrm{H}$ NMR (500 MHz, $\left.\mathrm{CDCl}_{3}\right) \square 8.44(\mathrm{~d}, J=8.4 \mathrm{~Hz}, 1 \mathrm{H}), 8.41(\mathrm{t}, J=6.1 \mathrm{~Hz}, 1 \mathrm{H}), 6.54(\mathrm{~d}, J=6.2 \mathrm{~Hz}$, $1 \mathrm{H}), 4.43(\mathrm{~m}, 1 \mathrm{H}), 4.06(\mathrm{~m}, 1 \mathrm{H}), 3.88-3.83(\mathrm{~m}, 2 \mathrm{H}), 3.35(\mathrm{dd}, J=5.2,16.6 \mathrm{~Hz}, 1 \mathrm{H}), 3.32-3.20$ (m, 1H), 2.95-2.90 (m, 1H), 2.30-2.22 (m, 2H), $1.18(\mathrm{~d}, J=7.0 \mathrm{~Hz}, 3 \mathrm{H}), 1.08(\mathrm{~d}, J=6.0 \mathrm{~Hz}$, 3H), $1.03(\mathrm{~d}, J=6.3 \mathrm{~Hz}, 3 \mathrm{H}) ;{ }^{13} \mathrm{C} \mathrm{NMR}\left(125 \mathrm{MHz}, \mathrm{CDCl}_{3}\right) \square$ 172.1, 172.0, 168.8, 75.6, 74.5, 
49.6, 44.8, 43.9, 42.8, 22.7, 20.5, 15.6; MS (positive FABMS) $272.3\left(\mathrm{M}^{+}+1\right)$; HRMS calcd for $\mathrm{C}_{12} \mathrm{H}_{22} \mathrm{~N}_{3} \mathrm{O}_{4}\left(\mathrm{M}^{+}+1\right)$ : 272.1610, found: 272.1617 . Compound 4: white solid, mp $234{ }^{\circ} \mathrm{C}(\mathrm{dec})$; $[\square]_{\mathrm{D}}=-89.0\left(c\right.$ 1.02, MeOH); IR (KBr) 3295, 1670, $1632 \mathrm{~cm}^{-1} ;{ }^{1} \mathrm{H}$ NMR (500 MHz, $\left.\mathrm{CDCl}_{3}\right) \square$ $8.56(\mathrm{t}, J=6.0 \mathrm{~Hz}, 1 \mathrm{H}), 8.28(\mathrm{~d}, J=8.1 \mathrm{~Hz}, 1 \mathrm{H}), 6.50(\mathrm{~d}, J=5.7 \mathrm{~Hz}, 1 \mathrm{H}), 4.39(\mathrm{~m}, 1 \mathrm{H}), 3.94(\mathrm{~m}$, $1 \mathrm{H}), 3.80(\mathrm{~m}, 1 \mathrm{H}), 3.76(\mathrm{dd}, J=6.7,16.6 \mathrm{~Hz}, 1 \mathrm{H}), 3.33(\mathrm{~m}, 1 \mathrm{H}), 2.92(\mathrm{dt}, J=2.2,12.9 \mathrm{~Hz}, 1 \mathrm{H})$, $2.47(\mathrm{dd}, J=4.0,12.6 \mathrm{~Hz}, 2 \mathrm{H}), 2.01(\mathrm{dd}, J=8.9,12.6 \mathrm{~Hz}, 1 \mathrm{H}), 1.18(\mathrm{~d}, J=6.9 \mathrm{~Hz}, 3 \mathrm{H}), 1.11(\mathrm{~d}$, $J=6.0 \mathrm{~Hz}, 3 \mathrm{H}), 0.93(\mathrm{~d}, J=6.2 \mathrm{~Hz}, 3 \mathrm{H}) ;{ }^{13} \mathrm{C} \mathrm{NMR}\left(125 \mathrm{MHz}, \mathrm{CDCl}_{3}\right) \square 172.2,170.6,168.9$, 67.4, 66.6, 47.8, 44.2, 44.0, 43.2, 18.8, 15.8, 15.1; MS (positive FABMS) $272.2\left(\mathrm{M}^{+}+1\right)$; HRMS calcd for $\mathrm{C}_{12} \mathrm{H}_{22} \mathrm{~N}_{3} \mathrm{O}_{4}\left(\mathrm{M}^{+}+1\right)$ : 272.1610, found: 272.1613 .

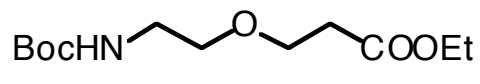

18

3-(2-tert-Butoxycarbonylaminoethoxy)propionic acid ethyl ester (18). Boc-protected ethanolamine 17 (Aldrich, $1.0 \mathrm{~g}, 6.2 \mathrm{mmol}$ ) in THF ( $8 \mathrm{~mL}$ ) was stirred with ca. $20 \mathrm{mg}$ of $\mathrm{Na}$ at room temperature. After $30 \mathrm{~min}$, ethyl acrylate $(0.93 \mathrm{~mL}, 9.3 \mathrm{mmol})$ was added, and stirring continued for $1 \mathrm{~h}$. The reaction was quenched with AcOH (2 drops) and concentrated. Flash chromatography (1:1 EtOAc/hexane) furnished the title compound $\mathbf{1 8}(1.32 \mathrm{~g})$ as an oil in $81 \%$ yield. IR (film) 3377, $1735 \mathrm{~cm}^{-1} ;{ }^{1} \mathrm{H}$ NMR (400 MHz, $\mathrm{CDCl}_{3}$ ) $\square 4.95$ (br s, $1 \mathrm{H}$ ), 4.16 (q, $J=7.2$ $\mathrm{Hz}, 2 \mathrm{H}), 3.72(\mathrm{t}, J=6.2 \mathrm{~Hz}, 2 \mathrm{H}), 3.51(\mathrm{t}, J=4.9 \mathrm{~Hz}, 2 \mathrm{H}), 3.29(\mathrm{~m}, 2 \mathrm{H}), 2.56(\mathrm{t}, J=6.2 \mathrm{~Hz}, 2 \mathrm{H})$, $1.44(\mathrm{~s}, 9 \mathrm{H}), 1.27(\mathrm{t}, J=7.2 \mathrm{~Hz}, 3 \mathrm{H}) ;{ }^{13} \mathrm{C} \mathrm{NMR}\left(100.6 \mathrm{MHz}, \mathrm{CDCl}_{3}\right) \square 172.0,156.3,79.6,70.3$, 66.6, 61.0, 40.6, 35.4, 28.8, 14.6; MS (EI) m/e $262\left(\mathrm{M}^{+}+1\right)$; HRMS calcd for $\mathrm{C}_{12} \mathrm{H}_{24} \mathrm{NO}_{5}\left(\mathrm{M}^{+}+1\right)$ : 262.1654, found: 262.1649 . 


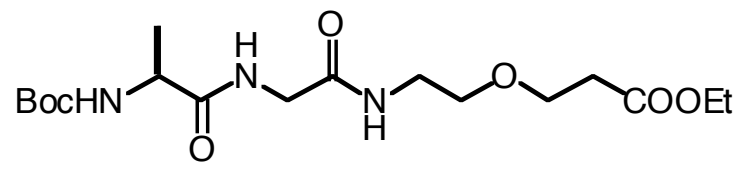

19

Boc-Ala-Gly-(4-oxa-Aca)-OMe (19). Oil; $[\square]_{\mathrm{D}}=-5.7$ (c 0.89, $\left.\mathrm{CHCl}_{3}\right)$; IR (film) 3317, $1660 \mathrm{~cm}^{-1} ;{ }^{1} \mathrm{H}$ NMR (400 MHz, $\mathrm{CDCl}_{3}$ ) $\square 7.23$ (br s, $\left.1 \mathrm{H}\right), 6.98$ (br s, $\left.1 \mathrm{H}\right), 5.47$ (d, J = $6.6 \mathrm{~Hz}$, $1 \mathrm{H}), 4.18(\mathrm{~m}, 1 \mathrm{H}), 4.13(\mathrm{q}, J=7.2 \mathrm{~Hz}, 2 \mathrm{H}), 3.99(\mathrm{dd}, J=5.8,16.7 \mathrm{~Hz}, 1 \mathrm{H}), 3.86(\mathrm{dd}, J=5.2$, $21.9 \mathrm{~Hz}, 1 \mathrm{H}), 3.68(\mathrm{t}, J=6.1 \mathrm{~Hz}, 2 \mathrm{H}), 3.51(\mathrm{t}, J=5.0 \mathrm{~Hz}, 2 \mathrm{H}), 3.48-3.32(\mathrm{~m}, 2 \mathrm{H}), 2.53(\mathrm{t}, J=$ $6.1 \mathrm{~Hz}, 2 \mathrm{H}), 1.40(\mathrm{~s}, 9 \mathrm{H}), 1.34(\mathrm{~d}, J=7.1 \mathrm{~Hz}, 3 \mathrm{H}), 1.24(\mathrm{t}, J=7.2 \mathrm{~Hz}, 3 \mathrm{H}) ;{ }^{13} \mathrm{C}$ NMR $(100.6$ $\left.\mathrm{MHz}, \mathrm{CDCl}_{3}\right) \square 173.8,172.4,169.4,156.1,80.4,69.5,66.4,61.1,50.8,43.3,39.5,35.2,28.7$, 18.7, 14.5; MS (positive FABMS) 390( $\left.\mathrm{M}^{+}+1\right)$; HRMS calcd for $\mathrm{C}_{17} \mathrm{H}_{32} \mathrm{~N}_{3} \mathrm{O}_{7}\left(\mathrm{M}^{+}+1\right)$ : 390.2240 , found: 390.2243 .

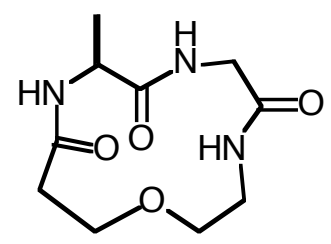

5

9S-Methyl-1-oxa-4,7,10-triaza-cyclotridecane-5,8,11-trione (5). White solid, mp 222$224^{\circ} \mathrm{C}(\mathrm{dec}) ;[\square]_{\mathrm{D}}=-97.5(c 0.75, \mathrm{MeOH}) ; \mathrm{IR}(\mathrm{KBr}) 3284,1676,1637 \mathrm{~cm}^{-1} ;{ }^{1} \mathrm{H}$ NMR $(500$ $\left.\mathrm{MHz}, \mathrm{CDCl}_{3}\right) \square 8.55(\mathrm{~d}, J=7.6 \mathrm{~Hz}, 1 \mathrm{H}), 8.43(\mathrm{t}, J=6.0 \mathrm{~Hz}, 1 \mathrm{H}), 6.65(\mathrm{~d}, J=4.3 \mathrm{~Hz}, 1 \mathrm{H}), 4.34$ (m, 1H), $3.81(\mathrm{dd}, J=7.1,16.5 \mathrm{~Hz}, 1 \mathrm{H}), 3.67-3.58(\mathrm{~m}, 3 \mathrm{H}), 3.39(\mathrm{dd}, J=5.4,16.5 \mathrm{~Hz}, 1 \mathrm{H}), 3.27$ $(\mathrm{td}, J=6.1,9.5 \mathrm{~Hz}, 1 \mathrm{H}), 3.19-3.05(\mathrm{~m}, 2 \mathrm{H}), 2.51(\mathrm{~m}, 1 \mathrm{H}), 2.19(\mathrm{dt}, J=3.0,14.4 \mathrm{~Hz}, 1 \mathrm{H}), 1.20$ $(\mathrm{d}, J=7.0 \mathrm{~Hz}, 3 \mathrm{H}) ;{ }^{13} \mathrm{C} \mathrm{NMR}\left(125 \mathrm{MHz}, \mathrm{CDCl}_{3}\right) \square 172.2,170.9,168.9,67.4,66.2,48.0,42.9$, 19.3, 15.8, 15.6; MS (positive FABMS) $244\left(\mathrm{M}^{+}+1\right)$; HRMS calcd for $\mathrm{C}_{10} \mathrm{H}_{18} \mathrm{~N}_{3} \mathrm{O}_{4}\left(\mathrm{M}^{+}+1\right)$ : 244.1297, found: 244.1285 . 


\section{References:}

(1) Kitagawa, O.; Vander Velde, D.; Dutta, D.; Morton, M.; Takusagawa, F.; Aubé, J. J. Am. Chem. Soc. 1995, 117, 5169-5178.

(2) Sato, M.; Kuroda, H.; Kaneko, C.; Furuya, T. Chem. Commun. 1994, 687-688.

(3) Sahasrabudhe, K.; Gracias, V.; Furness, K.; Smith, B. T.; Katz, C. E.; Reddy, D. S.; Aubé, J. J. Am. Chem. Soc. 2003, 125, 7914-7922.

(4) Crawley, G. C.; Briggs, M. T. J. Org. Chem. 1995, 60, 4264-4267.

(5) Danishefsky, S.; Kerwin, J. F. J. J. Org. Chem. 1982, 47, 1597-1598. 


\section{Some of the unsuccessful routes to cyclic compounds with aza-Aca-linkers:}
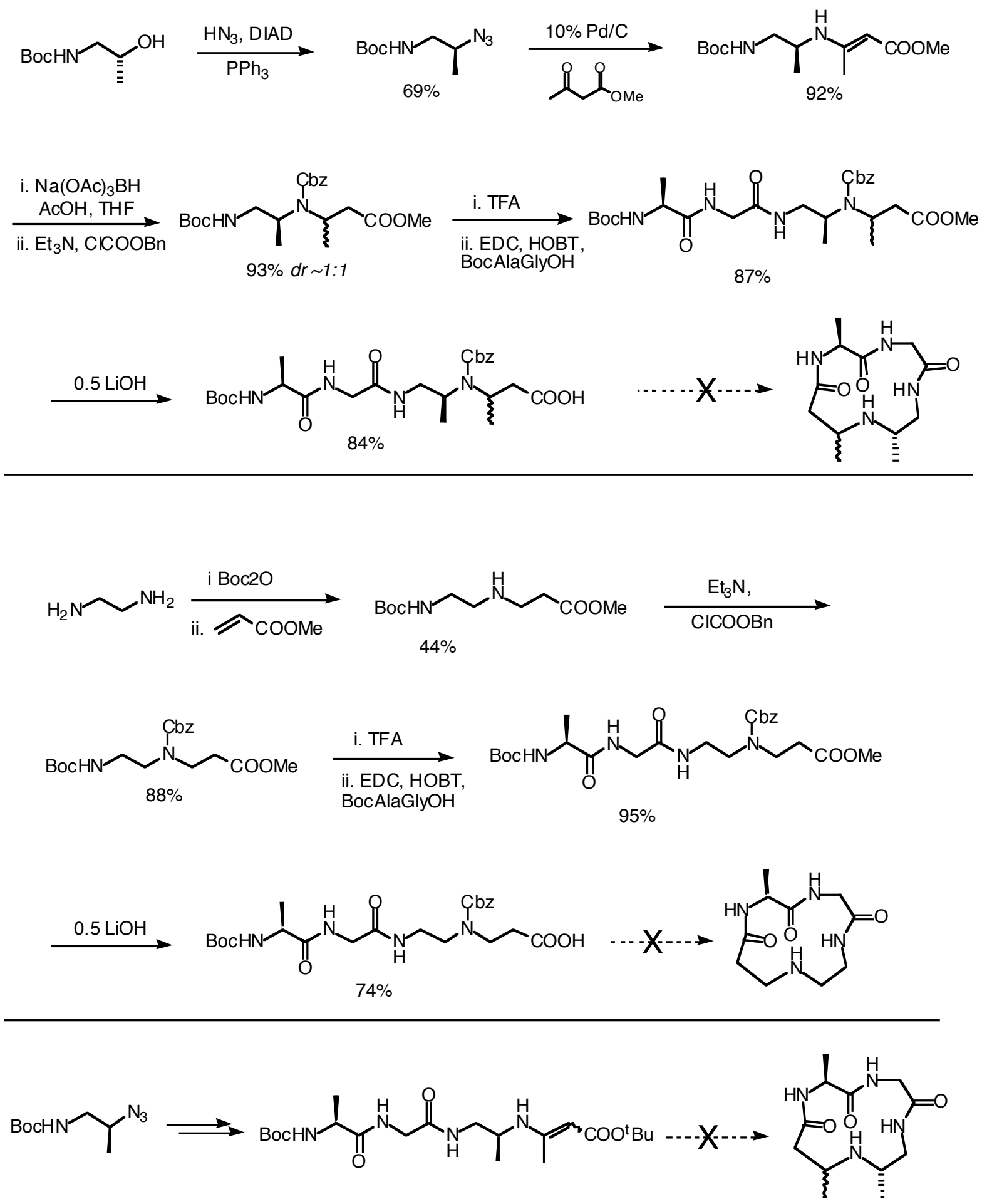


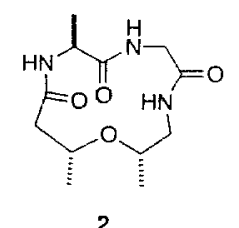

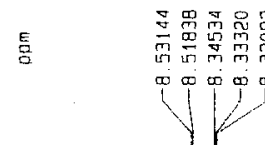

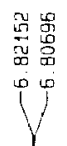

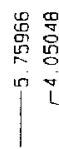

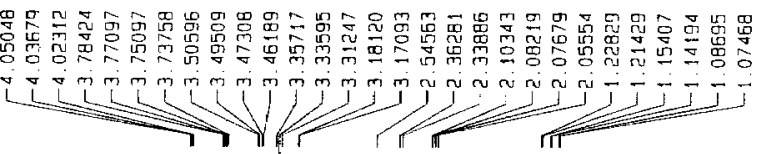

Current Data Parameters

NAME
EXPOO UST-II-133E,
PADCNO

Fa - acouisition Parameters

Catse - 20021217

$\begin{array}{ll}\text { TITE } & 16.16 \\ \text { INSTHLM } & \text { Snect }\end{array}$

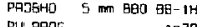

$\begin{array}{lr}\text { PULPROG } & 2970 \\ \text { TO } & 32768 \\ \text { SOL YENT } & \end{array}$

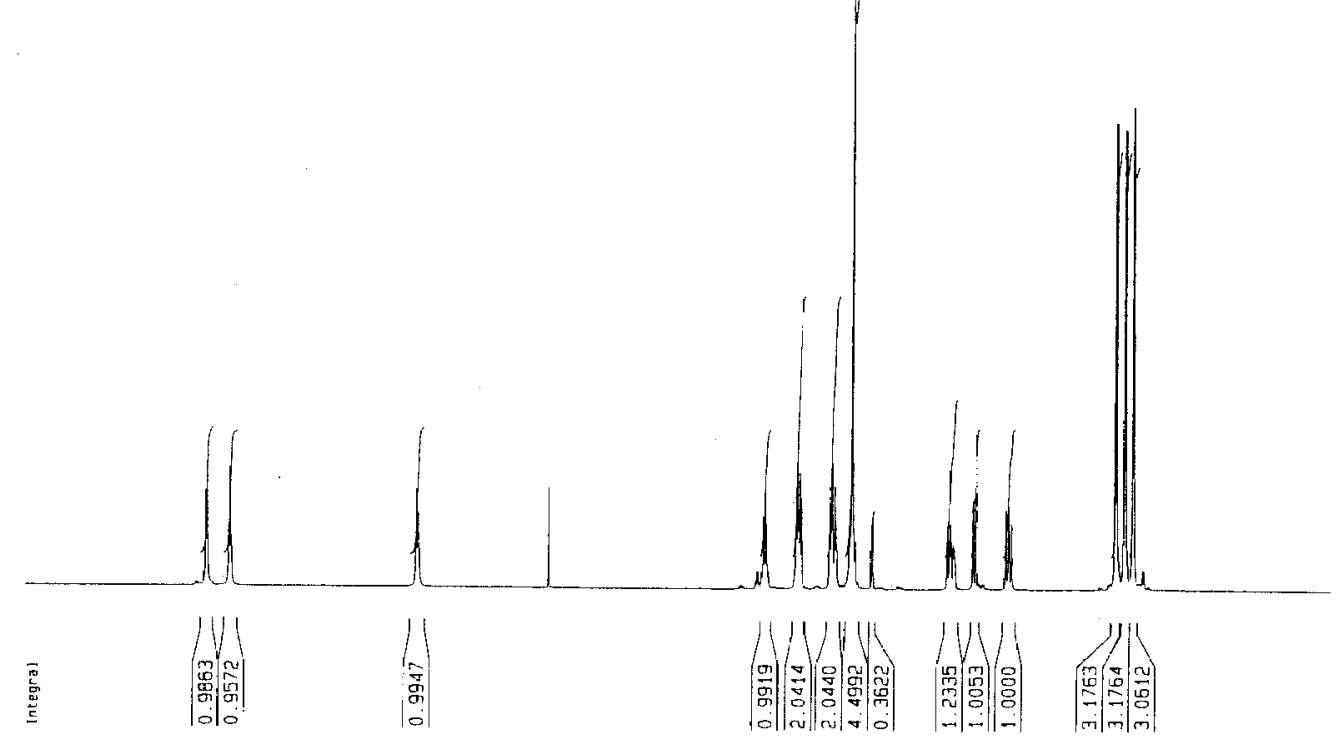

20
5

FIDFES $\quad 0.183399 \mathrm{~Hz}$

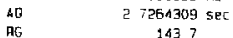

$\begin{array}{lr}\text { ON } & \quad 63.200 \text { uset } \\ \text { OE } & 6.00 \text { usec } \\ \text { TI } & 300.0 \mathrm{~K}\end{array}$

10. $093000000 \mathrm{sec}$

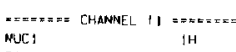

$\begin{array}{ll}\text { PH } & 7.70 \text { use } \\ \text { PLI } & -4.00 \mathrm{~dB}\end{array}$

SFOS $5001320005 \mathrm{MHz}$

21
25
25

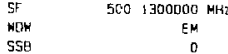

$\begin{array}{lc}\text { LA } & 0 \\ P B & 0.30 \mathrm{H} \\ \mathrm{PC} & 0\end{array}$

E1 - Processing ouranetors

51 Pi paramer

$\begin{array}{ll}\text { MC2 } & \text { GF } \\ 5 \text { FF } & 500.1300000 \mathrm{MHz}\end{array}$

$\begin{array}{lc}\text { NOM } & \text { OSINE } \\ \text { SSB } & 0 \\ \text { LB } & 0.30 \mathrm{~Hz}\end{array}$

10 NMP Giat parameters

Cx NMA biat porameters

$\begin{array}{ll}\mathrm{CX} & 20.00 \mathrm{~cm} \\ \mathrm{CH} & 2 \mathrm{CO}\end{array}$

Fi $\quad \begin{array}{ll}10.000 \mathrm{pom} \\ \mathrm{F}\end{array}$

$\begin{array}{ll}\mathrm{F}_{2} & -0.500 \mathrm{ppm} \\ \mathrm{Fz} & -250.07 \mathrm{~Hz}\end{array}$

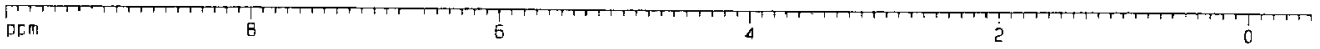

$0.52500 \mathrm{pPm} / \mathrm{cm}$
$2625 \mathrm{EBg} 24 \mathrm{~Hz} / \mathrm{Cm}$

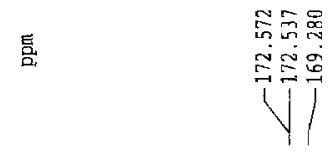

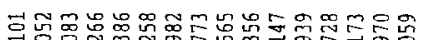

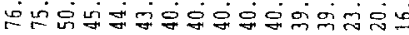

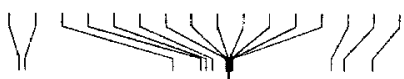

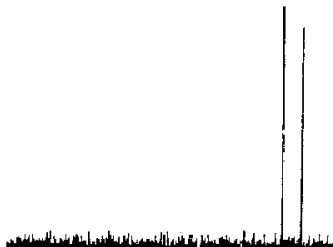

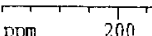

175

150

$+\frac{1}{125}$

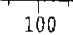

75

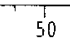

$\frac{1}{25}$

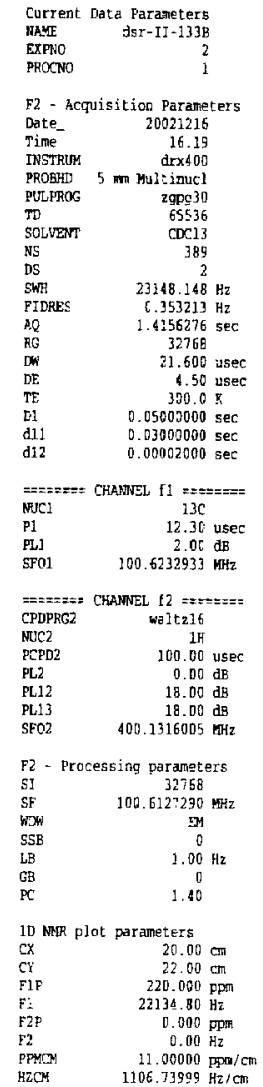



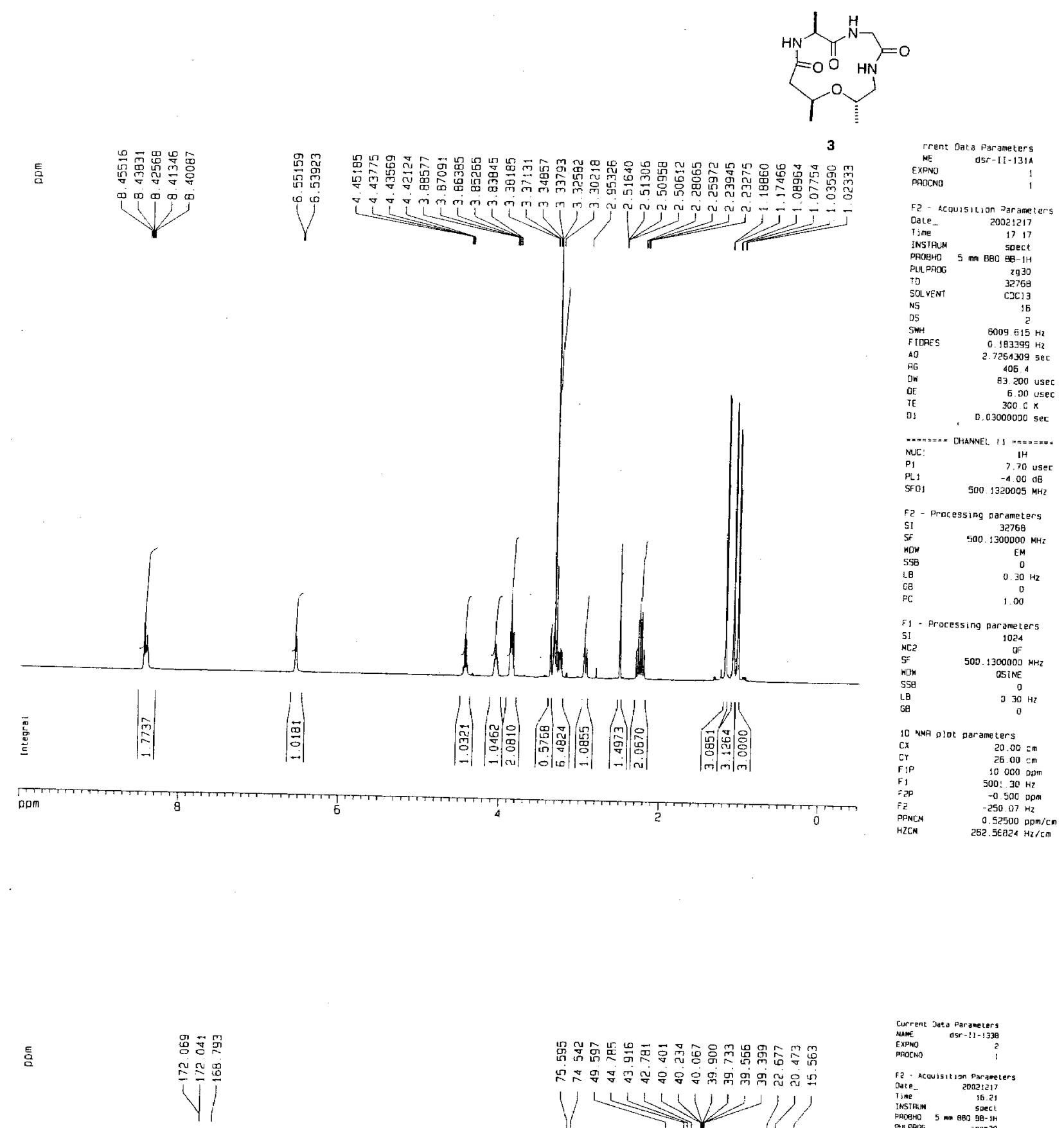

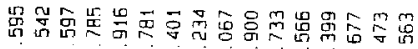

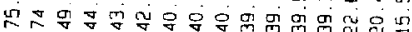
14 L 11
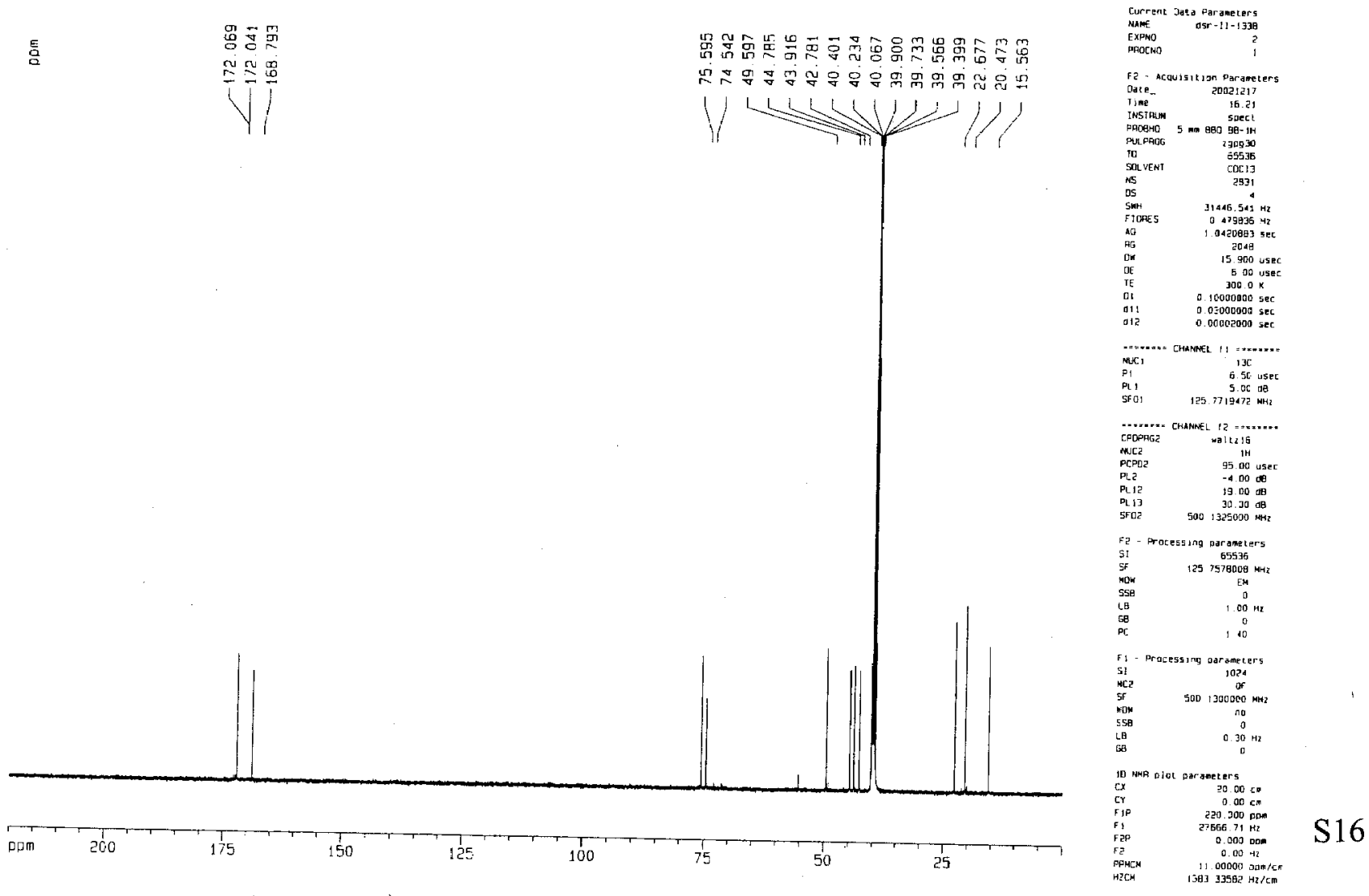

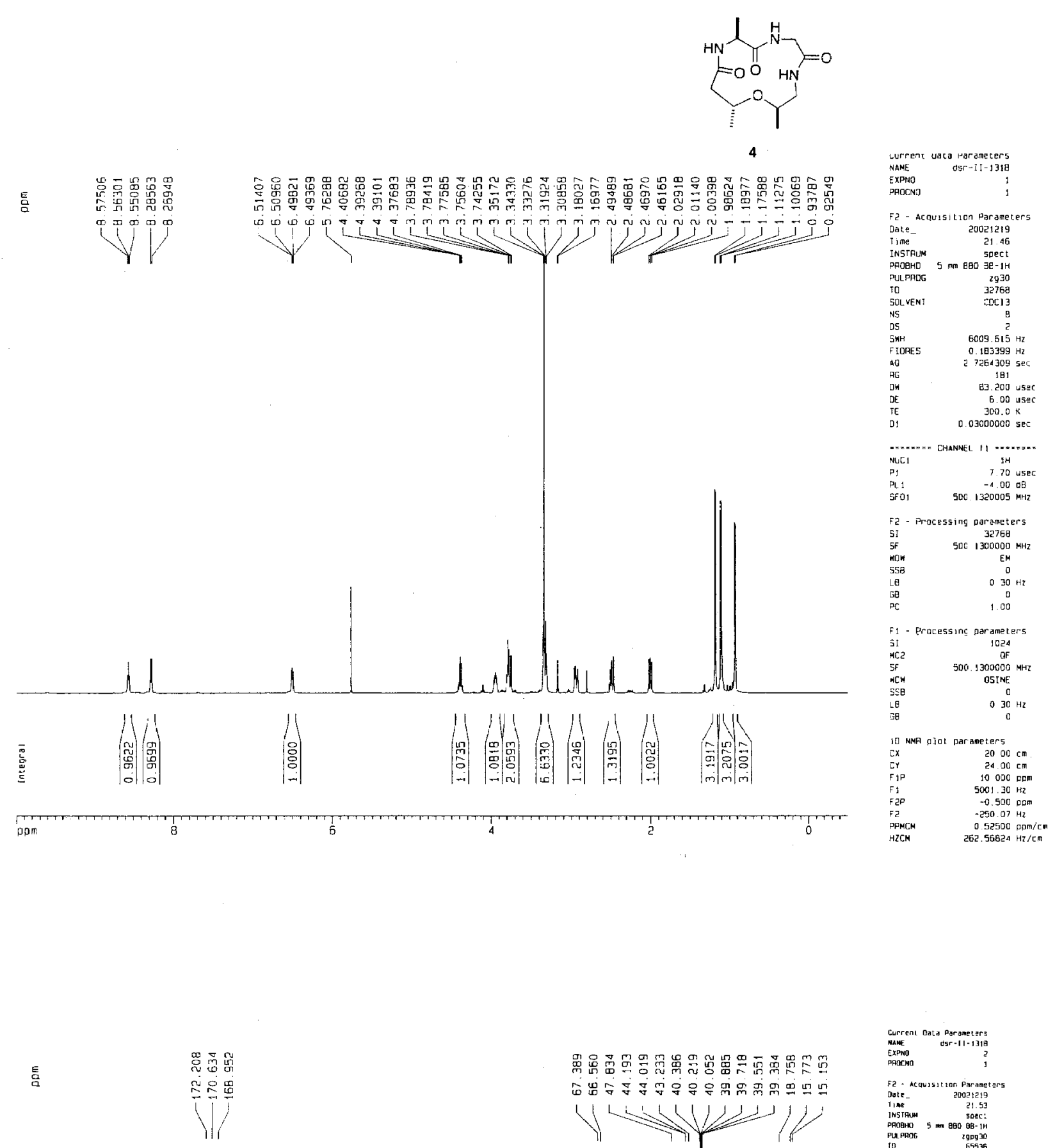

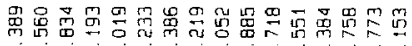

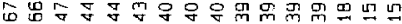

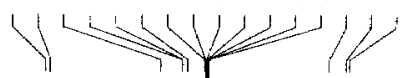

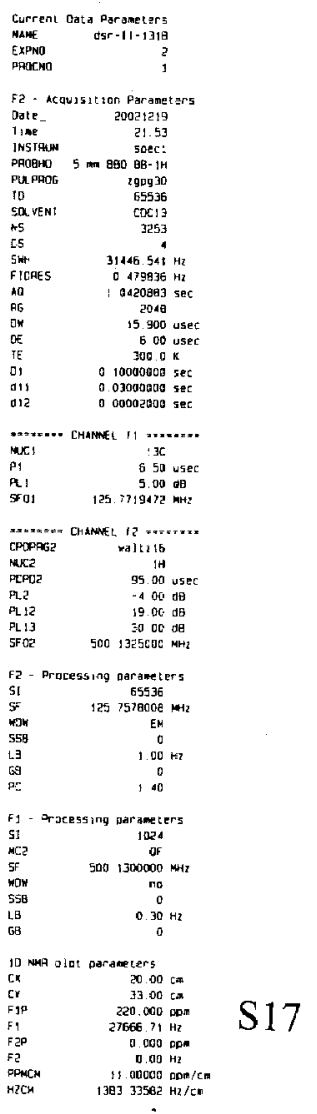




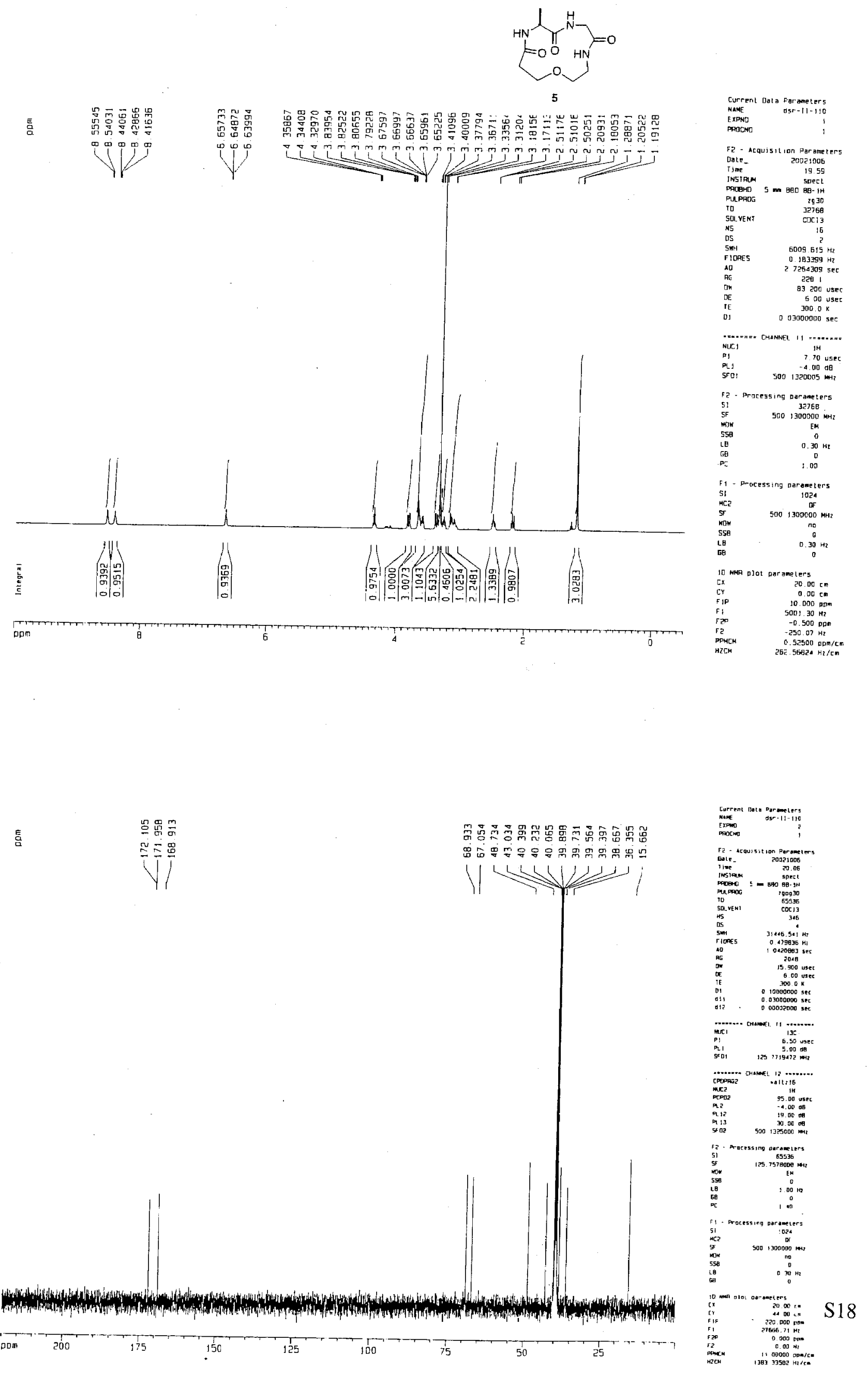




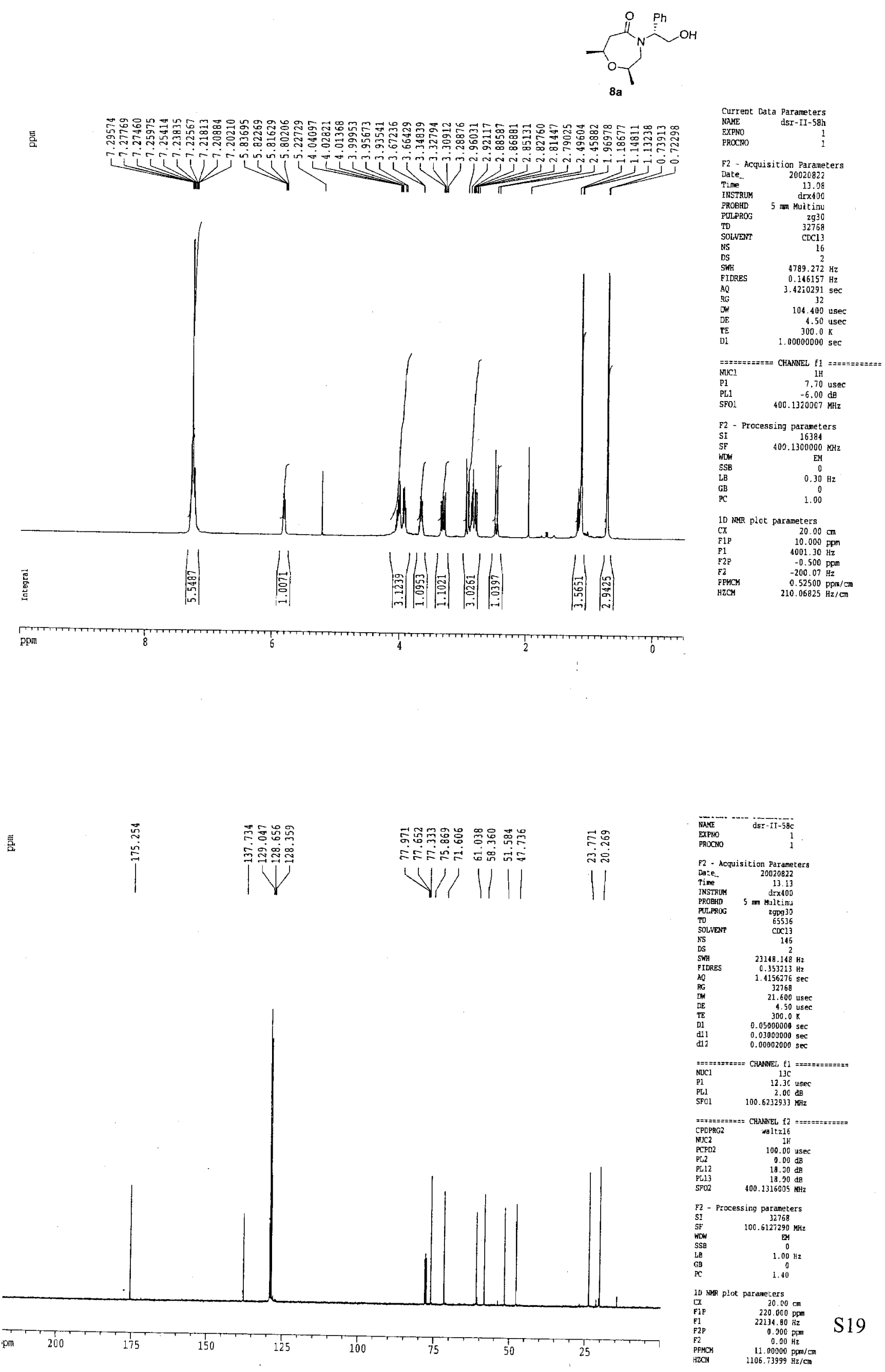




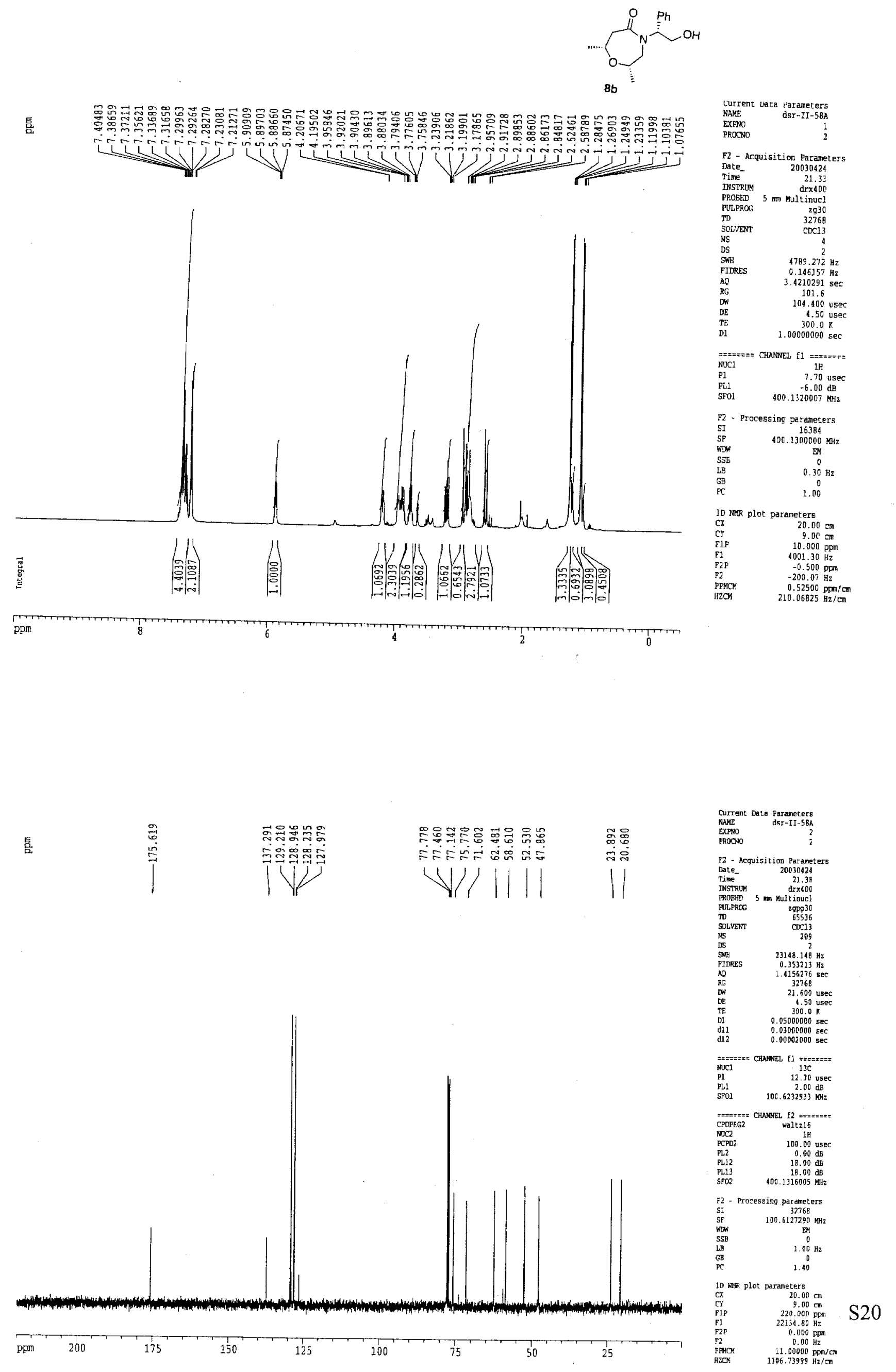



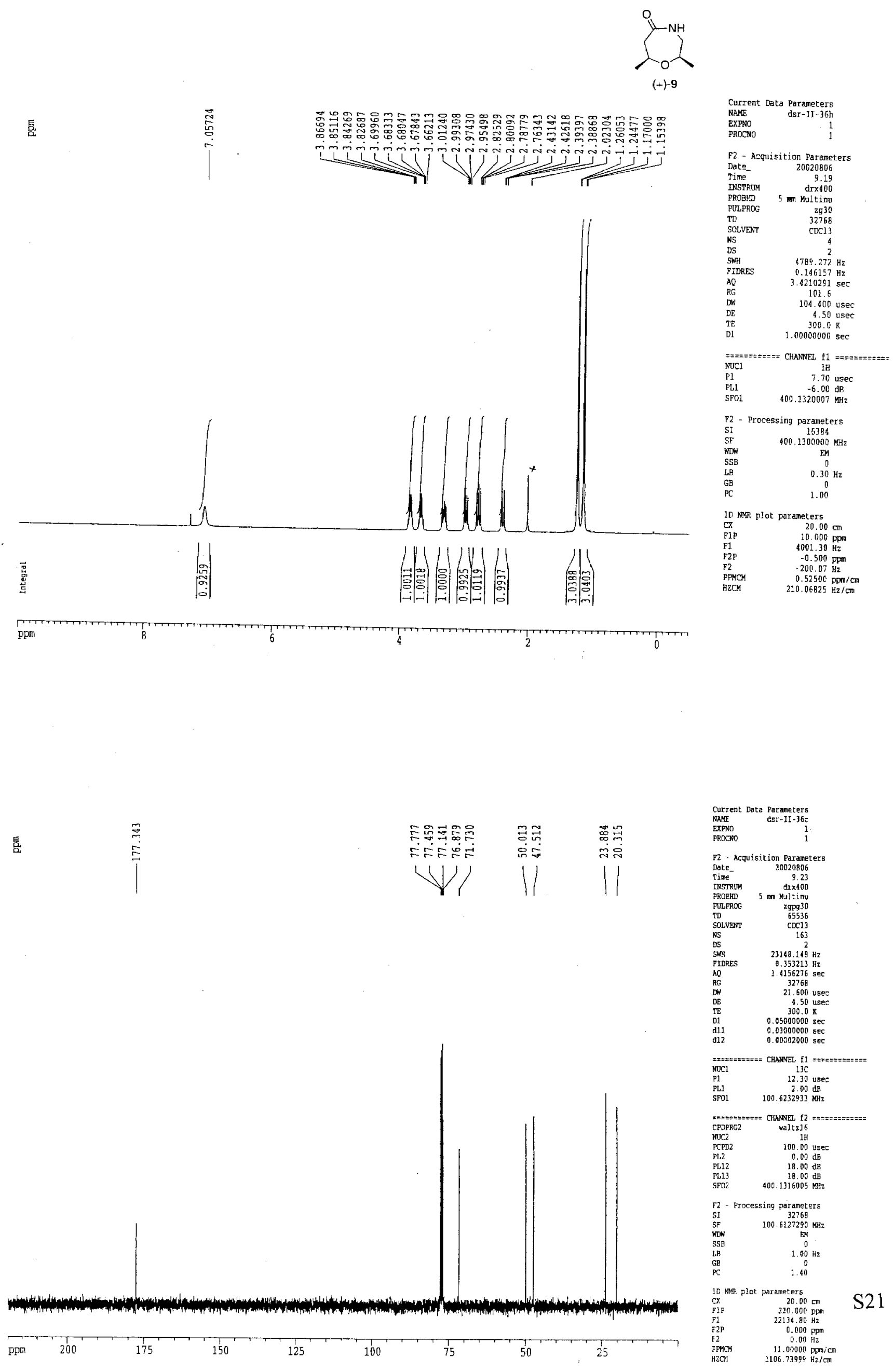

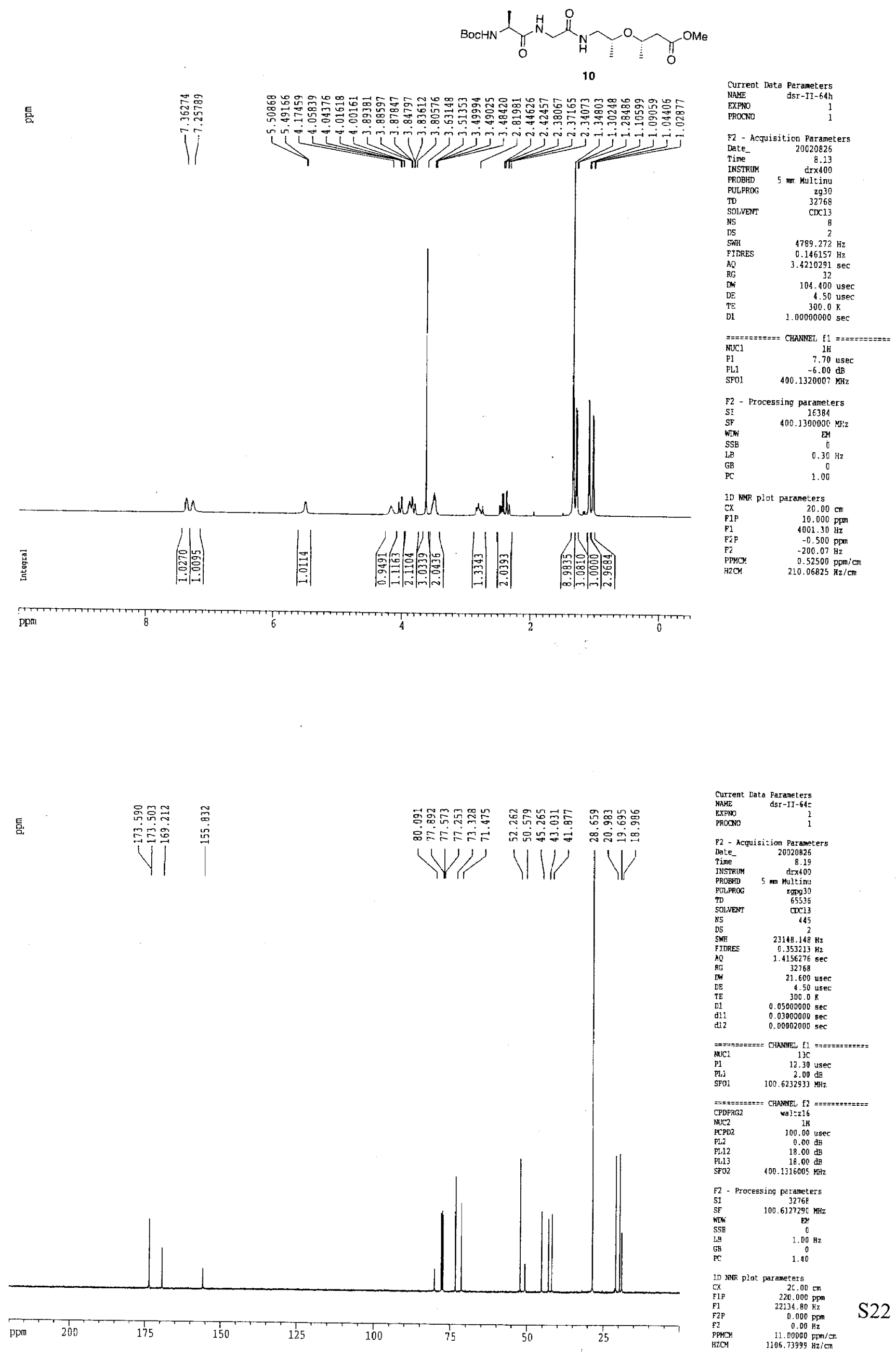


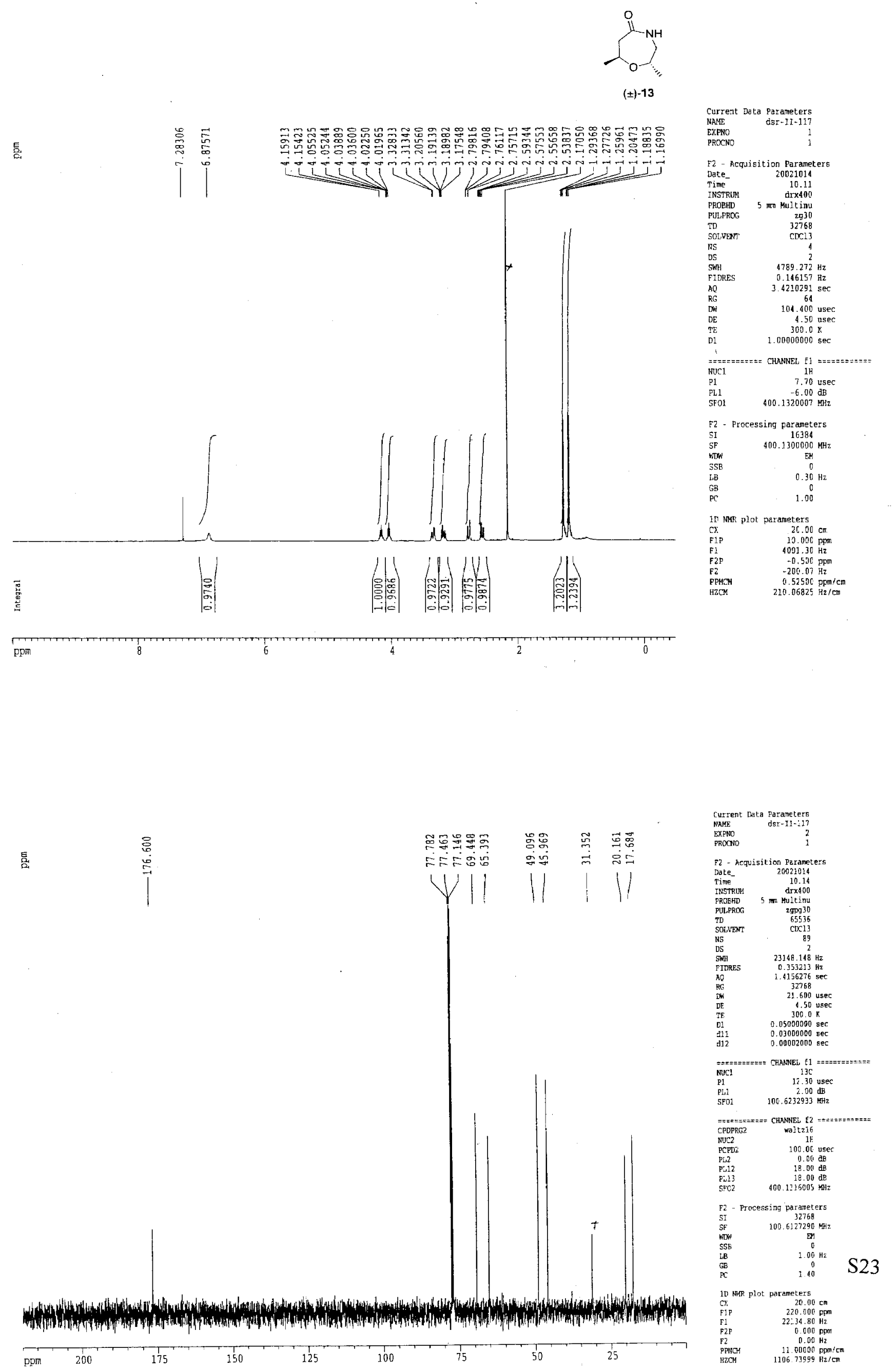




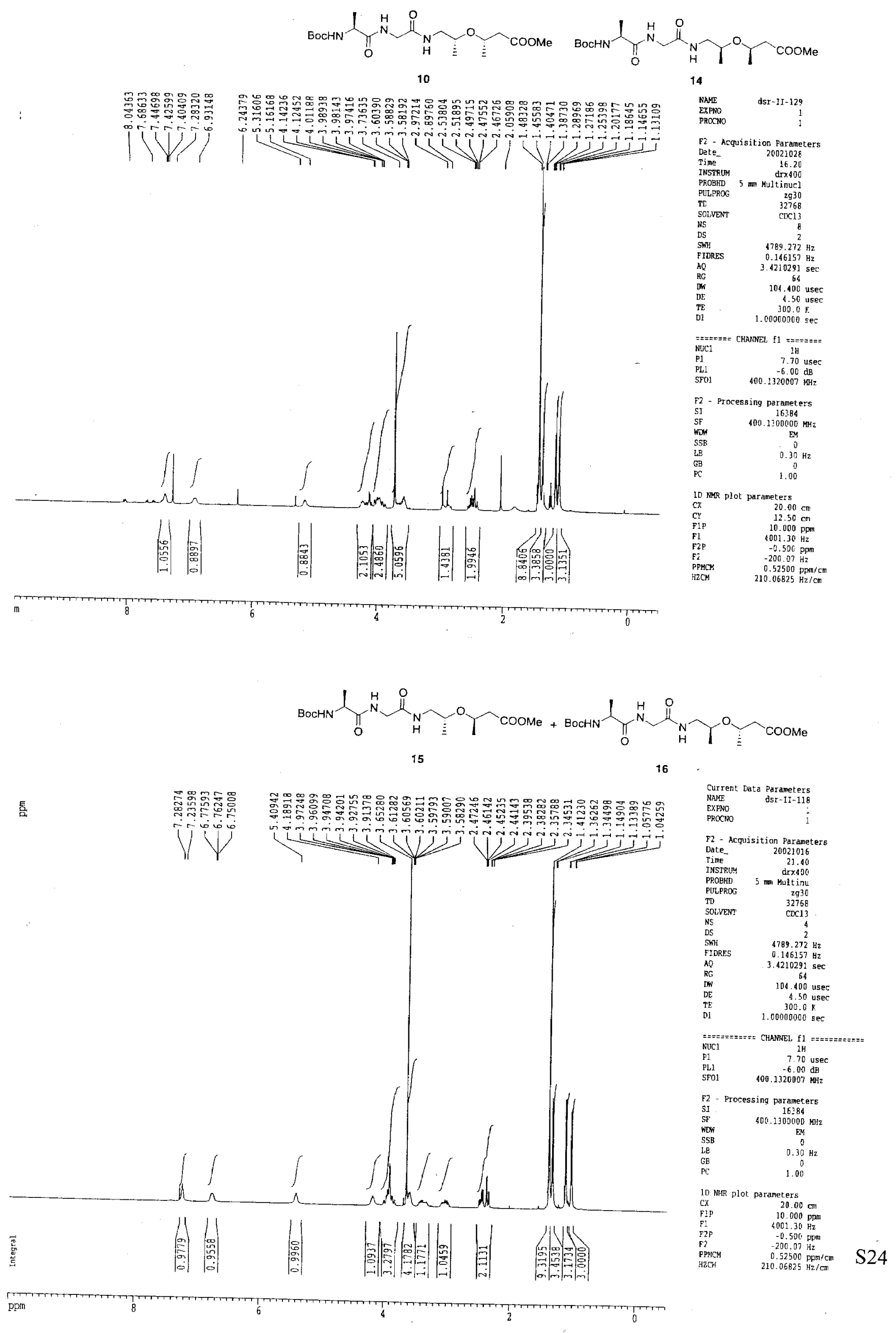


BocHN حO COOEI

18

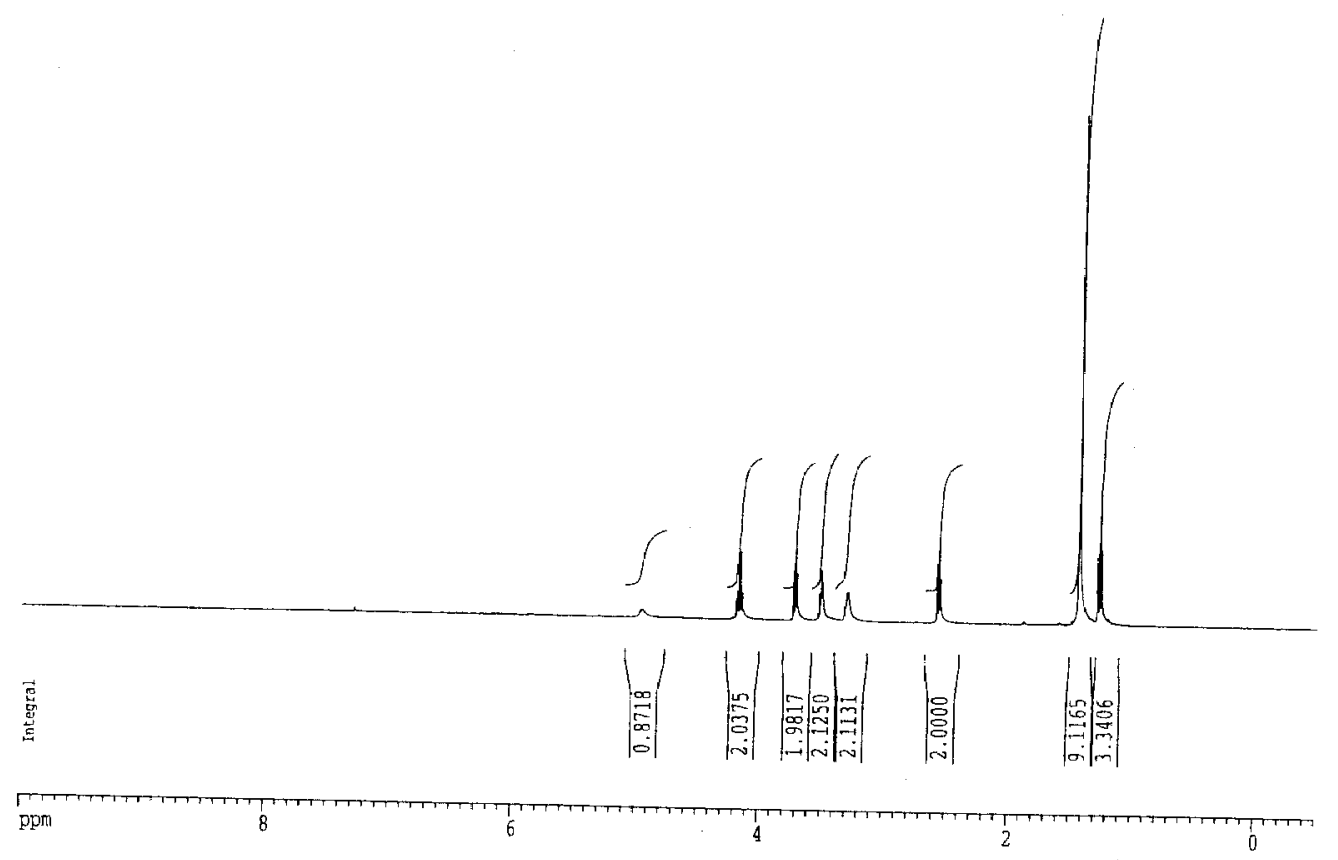

Current Data Paraineters

Exp:

PROCMO

1
1

F2 - Acquisitior. Parameters

Oate- 20030210

$\begin{array}{lr}\text { Time } & 26.38 \\ \text { INSTRIII } & \text { drx400 }\end{array}$

EROEHD 5 mm Muttinucl

SOL TDWT

NS

SWH $\quad 2$

FIDRES $\quad 0.145157 \mathrm{~Hz}$

${ }^{A D} \quad 3.4210291 \mathrm{sec}$

$\begin{array}{rr}R G & 64 \\ D W & 104.400 \text { usec } \\ D E & 4.50 \text { user }\end{array}$

$\begin{array}{lr}\mathrm{TE} & 300.0 \mathrm{~K} \\ \mathrm{DE} & 1.0000000 \mathrm{sec}\end{array}$

$===-s=$ CHANNEL, $\in 1$ =:ะ=:=:=

$\begin{array}{lr}\text { MuC1 } & \text { 1F: } \\ \text { P1 } & 7.7 C \text { บsec } \\ \text { PL1 } & -6.00 \text { diE } \\ \text { SF01 } & 400.1320007\end{array}$

F2 - Processing parametors

5I Processing pacaneters

$\begin{array}{ll}S F & 16384 \\ S F & 400.1300000 \mathrm{mHz}\end{array}$

SSE

$\mathrm{LB}$
$\mathrm{GB}$
$\mathrm{PC}$

EM
0

$\begin{array}{lr}\mathrm{PC} & 0 \\ \mathrm{PC} & 1.00\end{array}$

10 NMF plot parameters

CX plot parameters $20.00 \mathrm{~cm}$

$\begin{array}{lr}\text { F1P } & 8.00 \mathrm{~cm} \\ \mathrm{F1} & 10.000 \mathrm{ppm}\end{array}$

$\begin{array}{lr}\text { F1 } & 4001.30 \mathrm{~Hz} \\ \mathrm{~F} 2 \mathrm{P} & -0.500 \mathrm{ppm}\end{array}$

PPNCM $\quad-200.07 \mathrm{~Hz}$

$\begin{array}{lr}\mathrm{HECM} & 0.52500 \mathrm{ppt} / \mathrm{c} \\ & 210.06825 \mathrm{~Hz} / \mathrm{Cm}\end{array}$

镸

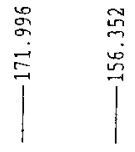

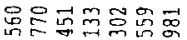

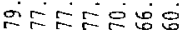

읭

தं

$\sqrt{0}$

L) 11
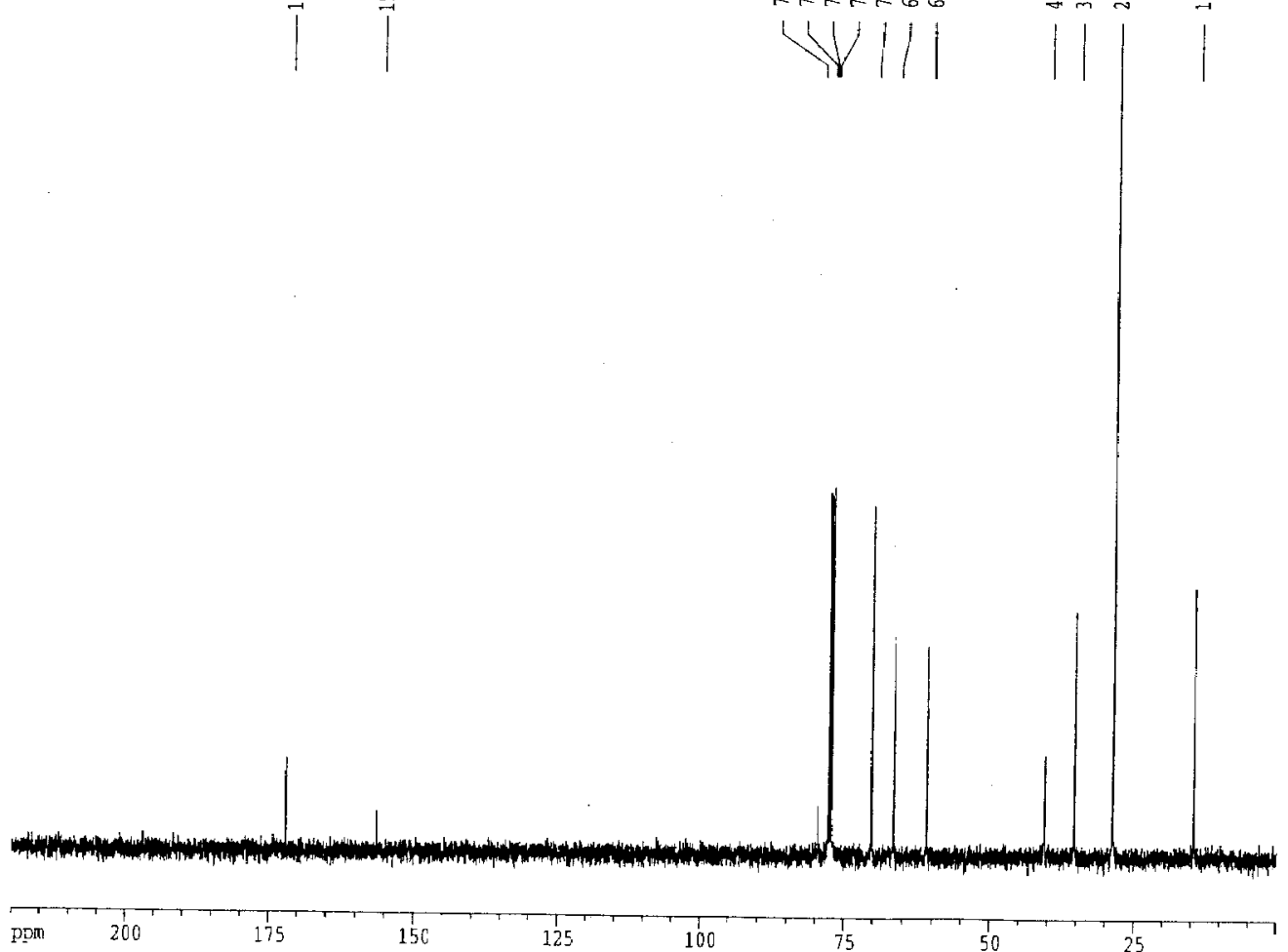

$T_{50}^{1}$
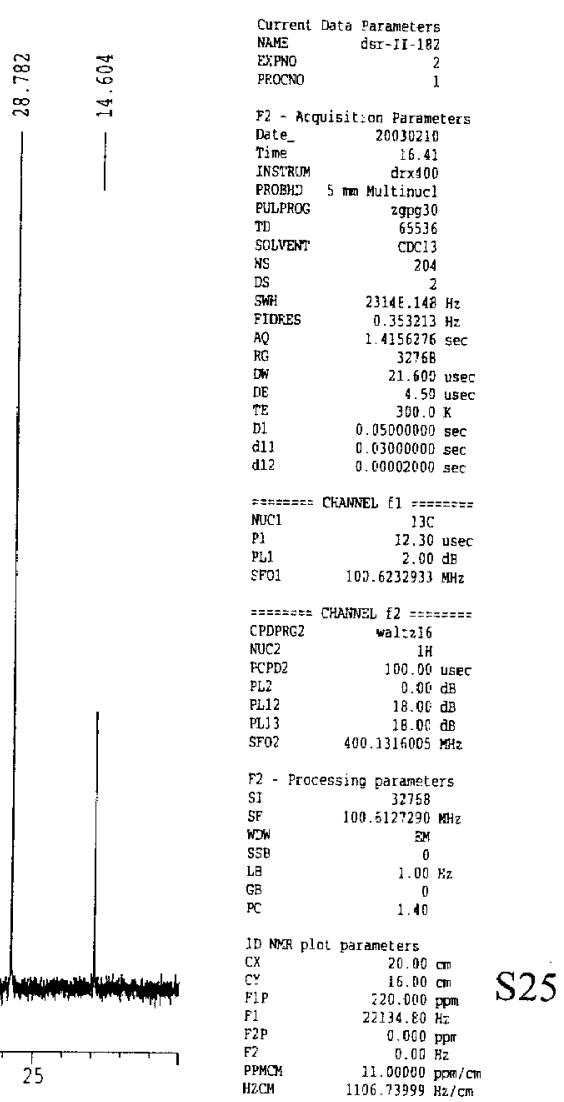

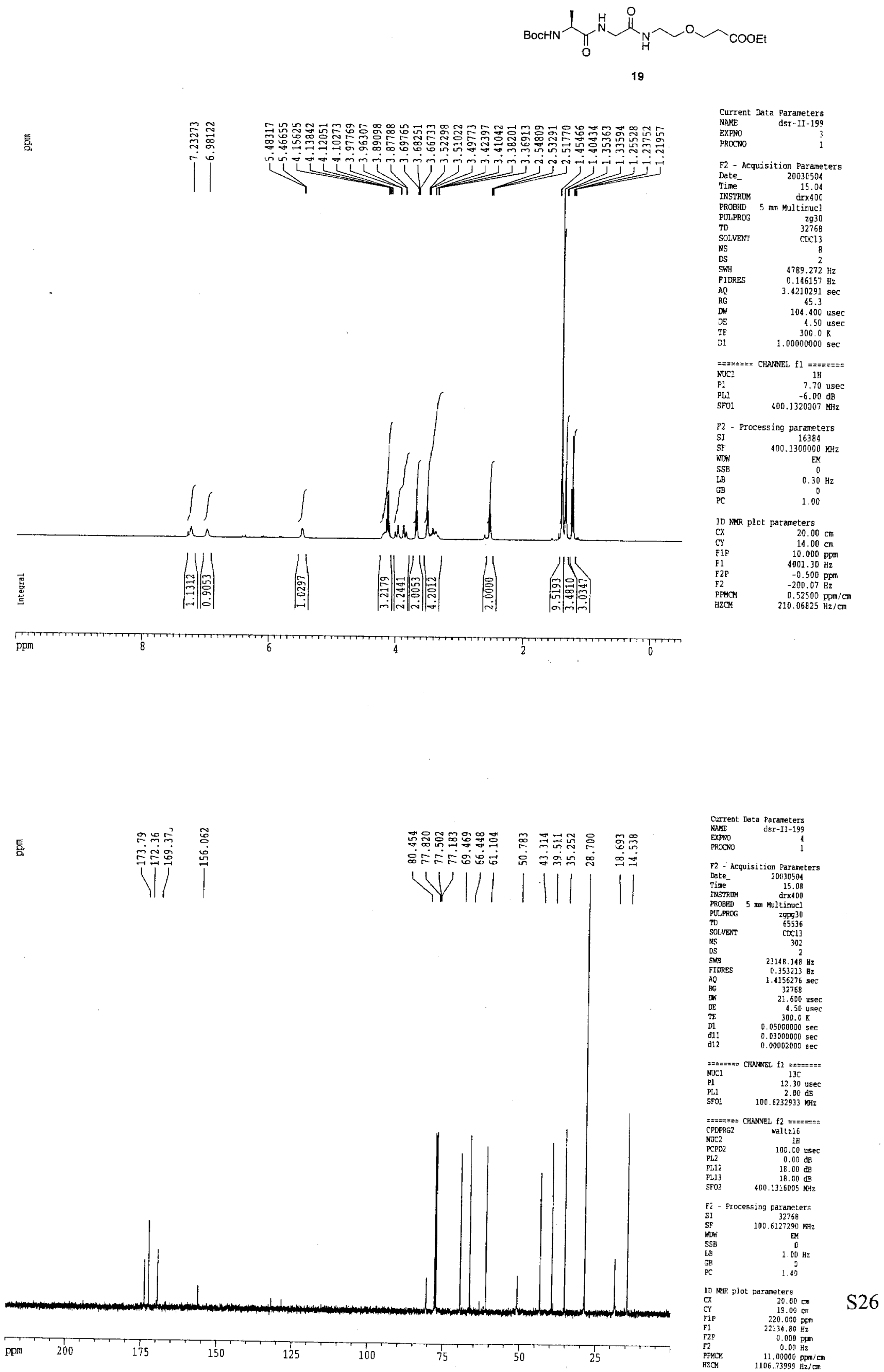